\title{
Two-dimensional adiabatic flows on to a black hole - I. Fluid accretion
}

\author{
Roger D. Blandford ${ }^{1 \star} \dagger$ and Mitchell C. Begelman ${ }^{2 \star} \ddagger$ \\ ${ }^{1}$ KIPAC, Stanford University, PO Box 20450, MS 29, Stanford, CA 94309, USA \\ ${ }^{2}$ JILA, University of Colorado, Boulder, CO 80309-0440, USA
}

Accepted 2003 November 13. Received 2003 October 22; in original form 2003 June 10

\begin{abstract}
When gas accretes on to a black hole, at a rate either much less than or much greater than the Eddington rate, it is likely to do so in an 'adiabatic' or radiatively inefficient manner. Under fluid (as opposed to magnetohydrodynamic) conditions, the disc should become convective and evolve toward a state of marginal instability. We model the resulting disc structure as 'gyrentropic', with convection proceeding along common surfaces of constant angular momentum, Bernouilli function and entropy, called 'gyrentropes'. We present a family of two-dimensional, self-similar models that describes the time-averaged disc structure. We then suppose that there is a self-similar, Newtonian torque, which dominates the angular momentum transport and that the Prandtl number is large so that convection dominates the heat transport. The torque drives inflow and meridional circulation and the resulting flow is computed. Convective transport will become ineffectual near the disc surface. It is conjectured that this will lead to a large increase of entropy across a 'thermal front', which we identify as the effective disc surface and the base of an outflow. The conservation of mass, momentum and energy across this thermal front permits a matching of the disc models to self-similar outflow solutions. We then demonstrate that self-similar disc solutions can be matched smoothly on to relativistic flows at small radius and thin discs at large radius. This model of adiabatic accretion is contrasted with some alternative models that have been discussed recently. The disc models developed in this paper should be useful for interpreting numerical, fluid dynamical simulations. Related principles to those described here may govern the behaviour of astrophysically relevant, magnetohydrodynamic disc models.
\end{abstract}

Key words: accretion, accretion discs - black hole physics - hydrodynamics - quasars: absorption lines.

\section{INTRODUCTION}

Recent X-ray observations of putative black holes in the nuclei of nearby elliptical galaxies show that they are extremely underluminous, some eight and four orders of magnitude below the Eddington and Bondi luminosities, respectively, (e.g. Di Matteo et al. 2000; Mushotzky et al. 2000; Baganoff et al. 2001). These observations have stimulated a fresh look at the nature of the accretion process. It appears that when the hole is 'underfed', specifically when the mass accretion rate is well below the fiducial Eddington rate, $\dot{m} \equiv \dot{M} / \dot{M}_{E} \ll 1$, where $\dot{M}_{E}=4 \pi G M / c \kappa$ (with $M$ denoting the black hole mass and $\kappa$ the relevant opacity), the radiative efficiency may be quite small, up to six orders of magnitude smaller than the traditional value $\sim 0.1 c^{2} \sim 10^{20} \mathrm{erg} \mathrm{g}^{-1}$.

\footnotetext{
^E-mail: rdb3@stanford.edu (RDB); mitch@jila.colorado.edu (MCB) $\dagger$ Also at: Theoretical Astrophysics, Caltech 130-33, Pasadena, CA 91125, USA.

$\ddagger$ Also at: Department of Astrophysical and Planetary Sciences, University of Colorado, Boulder, CO 80309-0440, USA.
}

One rationalization of this observation (e.g. Quataert \& Narayan 1999 and references therein), is that the gas accretes quite rapidly under the action of viscous stress but the dissipated energy is taken up almost exclusively by the ions, which do not radiate directly and which cannot heat the electrons efficiently. In a large number of recent publications, it has been supposed that electrons are heated minimally in this manner and that there is a conservative inflow in which hot ions advect essentially all of their binding energy across the black hole event horizon.

However, these 'advection-dominated accretion flow' (ADAF) solutions have a serious and fundamental shortcoming - the accreting gas is generically unbound and can escape to infinity. The reason why this happens is that the gas is likely to be supplied with sufficient angular momentum to orbit the hole and its inflow is controlled by the rate at which angular momentum is transported outward. This angular momentum transport, describable as a locally acting torque, is necessarily associated with a transport of energy. If we attempt to conserve mass, angular momentum and energy in the flow, we find that the Bernouilli function - the energy that the gas would have if it were allowed to expand adiabatically to infinity - is twice 
the local kinetic energy (Blandford \& Begelman 1999, henceforth BB99). In conventional accretion discs, this energy is radiated away and the gas remains bound. However, when cooling is unimportant to the inflow time - we call this case 'adiabatic' by analogy with the terminology for supernova remnants - something else must happen to the energy.

In an alternative description of adiabatic accretion, BB99 proposed that the inflow is non-conservative and that the radial energy transport drives an outflow that carries away mass, angular momentum and energy, allowing the disc to remain bound to the hole. (This was not a new proposal. Shakura \& Sunyaev (1973) were aware of this possibility and it has been discussed in many subsequent studies.) In these 'adiabatic inflow-outflow solutions' (ADIOS), the final accretion rate into the hole may be only a tiny fraction (in extreme cases $<10^{-5}$ ) of the mass supply at large radius (although this is not required). This leads to a much smaller luminosity than would be observed from a conservative flow. This is important from an observational perspective, because different assumptions concerning the extent and nature of the outflow affect the derived densities, temperatures, etc, of the emitting regions, and can lead to very different conclusions based on phenomenological fits to multiband data. In particular, most ADAF models posit essentially thermal emission, whereas ADIOS models are supposed to involve non-thermal emission by relativistic electrons as may be accelerated in the trans-sonic, shearing, magnetized flow surrounding the black hole. It has long been tempting (e.g. Rees et al. 1982; Blandford 1984) to associate underfed accretion on to very massive black holes with radio galaxies and quasars, and the presence of an outflow provides a natural agency for collimating relativistic jets, which are probably powered by electromagnetic or hydromagnetic processes close to the event horizon.

The epitome of an underfed black hole is the Galactic Centre (e.g. Melia \& Falcke 2001). Here the rate of gas supply at the Bondi radius $\left(r \sim 10^{5} \mathrm{~m}\right.$, where $\left.m \equiv G M / c^{2}\right)$ is estimated to be $\sim 10^{21} \mathrm{~g} \mathrm{~s}^{-1}$ (e.g. Di Matteo et al. 2000) while the bolometric luminosity is no more than $\sim 10^{36} \mathrm{erg} \mathrm{s}^{-1}$, giving an efficiency of conversion of mass supply to radiant energy of $\lesssim 10^{-6} c^{2}-$ hardly an advertisement for gravity power! Subsequent observations of Sgr A* have shown that the $\mathrm{X}$ ray emission is rapidly variable and has a steep spectrum (Baganoff et al. 2001), which is inconsistent with simple ADAF models that predicted a bremsstrahlung spectrum produced far from the black hole. Furthermore, Aitken et al. (2000) (cf. Bower et al. 2003) have measured millimetre linear polarization, which suggests that the plasma density close to the black hole is much less than would be associated with a conservative inflow (Agol 2000).

As pointed out in Begelman \& Meier (1982) and Blandford, Jaroszyński \& Kumar (1985) and BB99, the ADIOS analysis may also be appropriate for 'overfed' accretion, when $\dot{M} \gg \dot{M}_{E}$. Here the emissivity is large enough that radiation is emitted freely. However, the opacity is also large, so that the photons cannot escape on an inflow time-scale and are trapped by the flow. Hence the radiative efficiency, defined by the ratio of the escaping luminosity to the mass supply, is also low. At high accretion rates, the gas is again found to be unbound and it is proposed that inflow can take place only in the presence of a compensating outflow. There are also good observational reasons for believing that radiatively driven outflows are associated with overfed accretion. The Galactic source SS433 (Margon 1984; King, Taam \& Begelman 2000) appears to be an accreting black hole from which gas escapes at a rate at least a hundred times the critical rate. Galactic superluminal sources, such as GRS1915+112 (Mirabel \& Rodriguez 1999), also appear to be accreting rapidly and driving powerful outflows.
Overfed, massive holes have long been associated with radio-quiet quasars, which are classified as broad-absorption-line quasars when viewed from an equatorial direction (e.g. Blandford 1984; Weymann 1997). Although we do not understand enough physics to predict the maximum mass accretion rate for an underfed disc and the minimum one for the overfed case, the principle is clear - the classical, thin accretion disc is only a good description for a limited range of intermediate mass accretion rates and may only apply to a minority of accreting black holes.

There is now some observational evidence for the proposition that radiation-dominated accretion flows are also 'demand-limited' rather than 'supply driven'. An argument, originally due to Soltan (1982), associates the energy radiated by active galactic nuclei (AGN) (mostly quasars) with the mass of the relict black holes. The most recent estimate of these two quantities, allowing for the redshift of the emitted photons and the bolometric correction for unobserved emission (Yu \& Tremaine 2002), but see Fabian (2003), finds that they are in the ratio $\sim 0.1-0.2 c^{2}$. This implies that the binding energy of the accreting gas as it crosses the event horizon cannot be much less than $\sim 0.1$ as would be true of a radiation-dominated ADAF. Either black holes with $M \sim 10^{8} \mathrm{M}_{\odot}$, which account for most of the relict mass, acquire most of their mass during thin disc accretion, which requires an unlikely fine-tuning of the mass supply rate, or most of the mass supplied is blown away. [Note that this constraint does not require quasars to satisfy the Eddington limit (Begelman 2002).]

BB99 presented a family of simple, one-dimensional (1D) similarity solutions that span a large range of allowed flows, parametrized by the rates at which mass, angular momentum and energy are extracted. There was no discussion of the extraneous physical considerations that would allow one to determine these parameters within a broad range limited only by general thermodynamic and mechanical considerations. In this paper, we present a more detailed, fluid dynamical description of ADIOS discs that exhibits the manner by which the global transport of mass, angular momentum and energy might depend upon the microphysics assumed. We explicitly ignore magnetic field in this paper, so the solutions presented below are not directly applicable to observed discs but they are useful for bringing out salient principles. They may also aid in analysing numerical simulations.

In particular, we base our models on the prediction that adiabatic fluid discs are convective (Bardeen 1973; Begelman \& Meier 1982; Paczyński \& Abramowicz 1982; Blandford et al. 1985). In more recent developments, Quataert \& Narayan (1999) and Narayan, Igumenshchev \& Abramowicz (2000) have proposed that convection transports energy outward while carrying angular momentum inward (cf. Ryu \& Goodman 1992; Balbus 2000). This type of flow has been styled a convection-dominated accretion flow or 'CDAF'. In analytic models of a CDAF, the inward radial transport of angular momentum by convective motions exactly cancels the outward transport by viscous torque and the net mass accretion rate is very small.

In this paper, we adopt a quite different model for the convection. Following Bardeen (1973), Begelman \& Meier (1982), Paczyński \& Abramowicz (1982) and Blandford et al. (1985), we argue that the convective transport is vertical rather than radial, consistent with the Høiland criterion. When convection is efficient, it implies that the disc structure is 'gyrentropic' - that is to say the isentropes coincide with 'isogyres' (surfaces of constant specific angular momentum). This is the state of marginal, convective instability and it leads to the transport of mass, angular momentum and energy to the disc surface where these quantities can be removed by the 
outflow. This prescription allows us to compute two-dimensional (2D), hydrostatic disc models. However, these models do not describe inflow. We therefore add an explicit, though small, viscosity that leads to a torque across the gyrentropes. We show that this torque must also drive a meridional circulation, which can be computed. These circulating disc models are accurate only in the limit of small viscosity, when the convection can be efficient almost to the disc surface, just as happens in solar-type stars. The viscosity in accretion discs is not now thought to be so small and, as a consequence, the outflow can have a significant impact on the disc structure. We therefore further modify our circulation disc models by truncating them at a 'thermal front' where the convective energy flux is transformed into heat so that the associated pressure can self-consistently drive an outflow to infinity. In the case of an ion-dominated flow, the region of the disc downstream of the thermal front may be identified with an active corona.

One important difference between adiabatic accretion discs and their conventional, radiative counterparts is that, as they are thick, with opening angles $\sim 1$, the distinction between the thermal timescale and the viscous time-scale (e.g. Frank, King \& Raine 2001) is lost. The outflow adjusts on a time-scale $\sim O\left(\Omega^{-1}\right)$, where $\Omega$ is the angular frequency, whereas, as long as it is dynamical stable, the disc structure changes on a longer time-scale, $\sim O\left(\alpha^{-1} \Omega^{-1}\right)$, where $\alpha$ is the conventional viscosity parameter. These considerations will prove to be important when we discuss how a disc relaxes to a particular configuration in response to its assumed microphysical properties.

In the following section, we generalize the one-dimensional treatment of disc accretion in BB99 to accommodate alternative equations of state and introduce six models that span the types of flows that can be described by our solutions. In Section 3, we discuss two-dimensional convective stability in a rotating disc and derive models of two-dimensional gyrentropic discs in hydrostatic equilibrium. We next introduce a Newtonian, viscous stress that drives inflow and meridional circulation and supplies mass, angular momentum and energy to the disc surface (Section 4). Finally, these disc models are modified to match self-similar outflows (Section 5). A legitimate concern about self-similar disc models is that they may be invalid because they must fail at large and small radii. In Section 6, we demonstrate that our self-similar solutions can be matched on to a general relativistic flow close to the hole, and to a thin disc near an outer, transition radius. This is followed in Section 7 by a critical comparison with some alternative descriptions of adiabatic accretion that have appeared in the recent literature. We summarize our main conclusions in Section 8 . We shall discuss the more relevant problem of magnetic accretion and the application to selected astronomical sources in future papers.

\section{ONE-DIMENSIONAL DISCS}

\subsection{Conservation laws}

In BB99, we gave a simple explanation of why conservative, adiabatic accretion disc flows are unbound. Specifically, we showed, using a one-dimensional model, that the Bernouilli function, defined by

$B=H+\frac{\Omega^{2} R^{2}}{2}-\frac{1}{R}$,

equals $\Omega^{2} R^{2}$ when the total energy and angular momentum fluxes vanish. Here, $\Omega$ is the angular frequency, $H=\gamma P /(\gamma-1) \bar{\rho}$ is the enthalpy per unit mass, $P$ is the pressure, $\bar{\rho}$ is the density and $G M$ has been set to unity. Radiation-dominated and gas-dominated accretion correspond to adiabatic exponents $\gamma=4 / 3,5 / 3$, respectively. A positive Bernouilli function implies that an element of gas already has enough internal energy, after expanding adiabatically and doing work on its surroundings, to escape to infinity. Before we discuss possible mechanisms for effecting this removal, we must reprise and generalize the $1 \mathrm{D}$ results.

For convenience, we introduce an entropy function

$S=P^{1 / \gamma} / \bar{\rho}$,

which is monotonically related to the true thermodynamic entropy in thermal equilibrium. (We shall not require that the gas be in local thermodynamic equilibrium, only that $P \propto \bar{\rho}^{\gamma}$ when an element of gas changes its density in such a manner that there is negligible dissipation and heat exchange with its surroundings.)

For the moment we ignore vertical gradients, and suppose that the flow is stationary. (The assumption of stationarity need not seriously restrict our conclusions, if they are applied to the time-averaged flow.) The disc is hypothesized to evolve under a combination of internal torque and external loss of mass, angular momentum and energy. This allows the remaining gas to flow radially inward at a rate small compared with the rotational speed. In the absence of a better prescription and in order to elucidate general principles, we adopt, initially, a self-similar disc mass inflow

$\dot{M}=-\mathcal{M} V_{r} \propto R^{n} ; \quad 0 \leqslant n<1$,

where $\mathcal{M}$ is the mass per unit radius and $\mathcal{M}, V_{r}, n$ substitute for $\mu$, $-v, p$ of BB99, respectively. The reason for the restriction $n \geqslant 0$ is that gas is only supposed to leave the disc and for the inequality $n<$ 1 is that the energy flowing outward through the disc presumably decreases with radius as it is released mostly at small radius and is carried off by an outflow. We can also use equation (3) to define the mass loss per unit radius

$J \equiv \frac{\mathrm{d} \dot{M}}{\mathrm{~d} r}=n \frac{\dot{M}}{r}$,

where, and in what follows, we shall treat the disc and outflow as symmetric about the equatorial plane, so that all integral quantities refer to one hemisphere.

Angular momentum flows inward through the disc at a rate $F_{L}$. We suppose that angular momentum loss accompanies mass loss, and that

$\frac{\mathrm{d} F_{L}}{\mathrm{~d} \dot{M}}=(1+\eta) L$,

where $L=R^{2} \Omega$ is the specific angular momentum of the disc. Gas dynamical outflows that exert no reaction torque on the disc and simply carry off the angular momentum per unit mass of the gas at the start of the outflow have $\eta=0 . \eta$ measures the degree to which the outflowing angular momentum is either larger or smaller than this. If there are magnetic fields present, as we shall discuss explicitly in Paper II, then $\eta>0$ or if the outflow originates below the disc surface, as we discuss in Section 5, then $\eta<0$ and so we shall retain this generality. We assume that $F_{L}$ varies as a power law,

$F_{L}=\dot{M}(L-\mathcal{G})=\lambda \dot{M} R^{1 / 2}=\frac{2 n(1+\eta) \dot{M} L}{1+2 n}$,

where $\mathcal{G}$ ( $\equiv G$ in BB99) is the generalized, internal torque per unit $\dot{M}$ and is assumed to be positive. This equation shows how $\eta$ replaces the parameter $\lambda$ of BB99. As we discuss further below, equation (6) can be regarded as a definition of the torque but it has to be interpreted carefully in the presence of convection. 
Provided that this torque can be regarded as a local variable, then the second law of thermodynamics requires that it oppose the velocity shear (e.g. Landau \& Lifshitz 1959) so that

$0 \leqslant \eta<\frac{1}{2 n}$.

However, angular momentum transport need neither be local in this sense (e.g. Ryu \& Goodman 1992; Balbus 2000; Narayan et al. 2000; Quataert \& Chiang 2000) nor necessarily describable in the language of fluid mechanics (Quataert \& Gruzinov 2000). Furthermore, the first inequality in equation (7) is not strictly required when there is internal circulation, as we shall discuss below.

In a similar fashion, we assume a self-similar variation of the disc energy outflow and replace the energy parameter $\epsilon$ of BB99 with the dimensionless parameter $\beta$ according to

$F_{E}=\dot{M}(\mathcal{G} \Omega-B)=\frac{\epsilon \dot{M}}{R}=\frac{-n(\beta-1) \dot{M} B}{(1-n)}$.

The quantity $\dot{M} \mathcal{G} \Omega$ represents the mechanical work performed by the generalized torque from equation (6), while $\dot{M} B$ is the energy advected inward by the gas. With this definition of $\beta$, we find that

$\frac{\mathrm{d} F_{E}}{\mathrm{~d} \dot{M}}=(\beta-1) B$

in parallel to equation (5). There may be additional contributions to the energy flux, particularly associated with convection, hydromagnetic wave transport and thermal conduction. We shall include the first of these below.

We can combine equations (5) and (9) to obtain the useful relations

$\mathcal{G}=\left(\frac{1-n \beta}{1-n}\right) \frac{B R^{2}}{L}=\left(\frac{1-2 n \eta}{1+2 n}\right) L$.

Bound discs with $B<0, \mathcal{G}>0$ require a minimum energy outflow with

$\beta>\frac{1}{n}>1$.

In the limit of a thin disc, $B \rightarrow-1 / 2 R, L \rightarrow R^{1 / 2}$ and

$\beta \rightarrow \frac{3-4 n(1-n) \eta}{n(1+2 n)}$.

This additional, lower limit on $\beta$ imposes an additional, lower limit on $\eta$

$\eta>\frac{3-n(1+2 n) \beta}{4 n(1-n)}$.

In simple, fluid models with $\eta=0$,

$\beta \geqslant \beta_{\min } \equiv \frac{3}{n(1+2 n)}$.

As explained in BB99, the self-similar scalings for pressure and density are $P \propto R^{n-5 / 2}, \bar{\rho} \propto R^{n-3 / 2}$, which transform the approximate radial equation of motion into the form

$\Omega^{2} R^{2}-\frac{1}{R}+(5 / 2-n) \frac{P}{\bar{\rho}}=0$.

Combining with equation (1), we obtain

$L=\left\{\frac{2(5-2 n)[1-(\gamma-1) B R]-2 \gamma(3-2 n)]}{5-2 n+\gamma(2 n-1)}\right\}^{1 / 2} R^{1 / 2}$

Combining equation (16) with equation (10) allows us to solve for $L, B, \mathcal{G}$ as explicit functions of $n, \beta, \eta$ subject to inequalities (3), (7), (11) and (13).
However, in this paper we shall follow a different approach. Instead of emphasizing the full range of outflow models that might be possible, we shall explore the manner in which the local physics might dictate a particular, global solution. It is then more convenient to express $n, \beta, \eta$ in terms of the fluid variables that describe the disc. A convenient, general choice is $L, B, \mathcal{G}$ and we find that

$n(L, B)=\frac{(5-\gamma) L^{2} / R+10(\gamma-1) B R+2(3 \gamma-5)}{2(\gamma-1)\left(2+2 B R-L^{2} / R\right)}$

independent of $\mathcal{G}$. Lengthier expressions can be derived for $\beta(L, B, \mathcal{G})$ and $\eta(L, B, \mathcal{G})$, which will not be reproduced. For the special case of interest here, $\eta=0$, it is simplest to solve equation (10) for $\beta$

$\beta(L, B)=\frac{(1+2 n) B R-(1-n) L^{2} / R}{n(1+2 n) B R}$,

and substitute equation (17). In this case, $\beta$ is also independent of $\mathcal{G}$.

\subsection{Illustrative models}

In this paper we shall use six examples of combined disk-wind flows to allow us to explore a range of models described by our approach and which illustrate some more general principles. We introduce here the one-dimensional versions of these models, which we shall shortly describe in two dimensions. The principal 1D and 2D characteristics of these models are listed in Table 1 and plotted for four of these models, with $\eta=0$ and $\gamma=5 / 3$, in Fig. 1.

\subsubsection{Model I: fiducial, ion-supported disc}

This reference model assumes that the hole is underfed so that the gas pressure is ion-dominated (with $\gamma=5 / 3$ ). We suppose that the gas has moderate pressure (parametrized by $B=-0.15 / R$ ) and centrifugal support (parametrized by $L=0.75 R^{1 / 2}$ ) and consequently moderate mass and energy loss rates $(n \sim 0.6, \beta \sim 3)$.

\subsubsection{Model II: radiation-supported disc}

Overfed, radiation-dominated accretion with $\gamma=4 / 3 . L, B$ are chosen to give similar values of $n, \beta$ to model I.

\subsubsection{Model III: thick disc}

A much less bound version of model I $(B \sim-0.03 / R)$ but with a similar circular velocity. The $2 \mathrm{D}$ disc is very thick with a small opening angle, $\theta \sim 0.2$. The mass-loss rate is high $(n \sim 0.9)$ and so the energy loss parameter is low $(\beta \sim 1.7)$.

\subsubsection{Model IV: intermediate disc}

A more tightly bound version of model $\mathrm{I}(B=-0.25 / R)$, with a somewhat thinner disc. The mass-loss rate is relatively small $(n \sim$ 0.2 ) and so $\beta \sim 14$ has to be correspondingly large to compensate.

\subsubsection{Model V: fast disc}

A fast, thin $\operatorname{disc}\left(L \sim 0.99 R^{1 / 2}\right)$. In order to create a model to explore this limit we choose to keep the mass-loss rate index $n$ equal to that of model I. 
Table 1. Physical parameters that characterize the six disk-outflow models, identified by Roman numerals. The models are described in four formalisms of increasing sophistication: a 1D model, a 2D gyrentropic model extending to zero pressure and density, the same with the internal circulation and convective heat flux computed self-consistently (Circ.), and a disc where the surface is chosen to be a thermal front that coincides with the base of the outflow (Out). The models are parametrized by the specific heat ratio $\gamma$, the equatorial Bernouilli function $b_{0}(=R B$ for $1 \mathrm{D}$ models) and the scaled, equatorial angular momentum $\ell_{0}\left(=R^{-1 / 2} L\right.$ for $1 \mathrm{D}$ models). These parameters are sufficient to derive the mass loss exponent $n$ and the energy loss exponent $\beta$ in 1D, assuming that $\eta=0$. The basic $2 \mathrm{D}$ treatment adds a disc opening angle $\theta_{d}$. A viscous torque is introduced in the circulation models, which modifies the derived energy loss rate. Further modifications to $\theta_{d}, \beta, \eta$ occur in the outflow models. The scaled Bernouilli function changes from $b_{d}$ to $b_{\mathrm{w}}$ on crossing the thermal front (at an angle $\theta_{\mathrm{w}}$ ) in the outflow models. Asymptotically, the wind flows on the surface of an evacuated cone with scaled speed $v_{\infty}$ and opening angle $\theta_{j}$.

\begin{tabular}{|c|c|c|c|c|c|c|c|c|c|c|c|c|c|c|}
\hline $\begin{array}{l}\text { All } \\
\text { No. }\end{array}$ & $\gamma$ & $\ell_{0}$ & $b_{0}$ & $\begin{array}{c}1 \mathrm{D} \\
n\end{array}$ & $\beta$ & $\begin{array}{l}2 \mathrm{D} \\
\theta_{d}\end{array}$ & $\begin{array}{c}\text { Circ. } \\
\beta\end{array}$ & $\begin{array}{c}\text { Out. } \\
\beta\end{array}$ & $\eta$ & $b_{d}$ & $\theta_{\mathrm{w}}$ & $\begin{array}{c}\text { Wind } \\
b_{\mathrm{w}}\end{array}$ & $v_{\infty}$ & $\theta_{j}$ \\
\hline I & 1.67 & 0.75 & -0.15 & 0.58 & 3.01 & 0.42 & 2.86 & 3.27 & -0.10 & -0.31 & 0.64 & 0.62 & 0.77 & 0.39 \\
\hline II & 1.33 & 0.90 & -0.20 & 0.58 & 3.12 & 0.60 & 2.87 & 3.26 & -0.09 & -0.30 & 0.91 & 0.59 & 0.89 & 0.39 \\
\hline III & 1.67 & 0.75 & -0.03 & 0.91 & 1.74 & 0.18 & 1.54 & 1.69 & -0.03 & -0.19 & 0.36 & 0.11 & 0.19 & 0.32 \\
\hline IV & 1.67 & 0.75 & -0.25 & 0.17 & 14.4 & 0.56 & 13.9 & 17.6 & -0.76 & -0.37 & 0.77 & 5.60 & 2.74 & 0.66 \\
\hline V & 1.67 & 0.99 & -0.46 & 0.58 & 2.38 & 1.34 & 2.33 & 2.59 & -0.14 & -0.49 & 1.40 & 0.55 & 1.13 & 1.34 \\
\hline VI & 1.67 & 0.63 & -0.02 & 0.58 & 10.0 & 0.11 & 7.05 & 8.25 & -0.09 & -0.11 & 0.34 & 0.78 & 0.47 & 0.24 \\
\hline
\end{tabular}

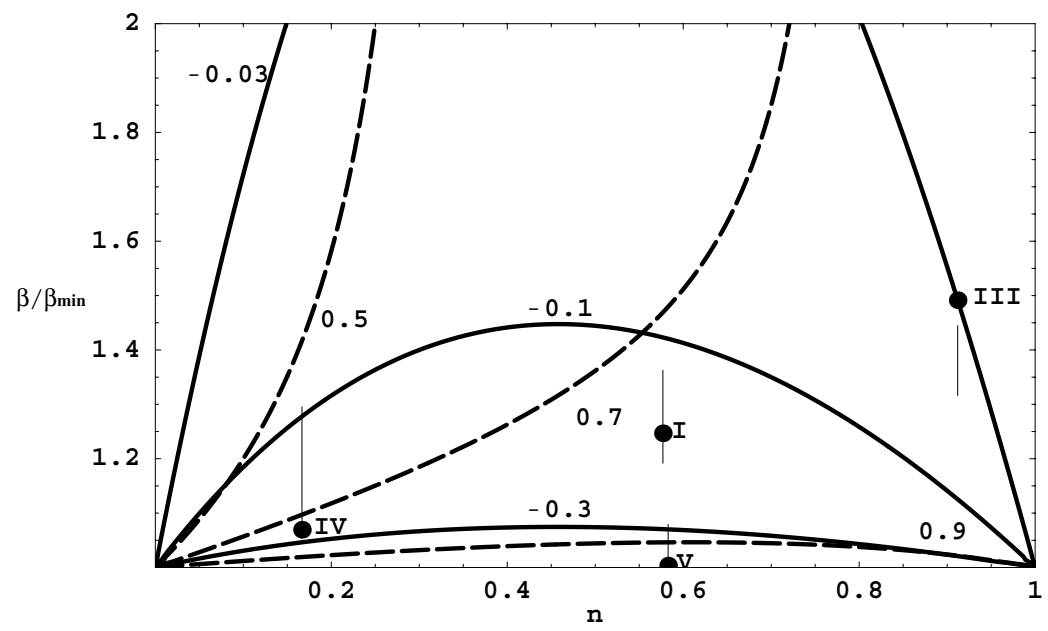

Figure 1. Four disc models of self-similar, gyrentropic accretion discussed in the text and located on the $n-\beta / \beta_{\min }$ plane assuming a fluid disc with $\eta=0$, $\gamma=5 / 3$. (Model II has $\gamma=4 / 3$; model VI has large $\beta$.) The ordinate is the ratio of the scaled energy outflow to that required for a thin disc with $L=R^{1 / 2}$, $B=-0.5 / R$. The dashed lines are contours of constant $L / R^{1 / 2}$ in the case of $1 \mathrm{D}$ models and $\ell_{0}$ for $2 \mathrm{D}$ models; the solid lines are contours of constant $B R$ for $1 \mathrm{D}$ models and $b_{0}$ for $2 \mathrm{D}$. The points correspond to the values of $\beta / \beta_{\min }$ for $1 \mathrm{D}$ models. The vertical lines passing upward through these points connect the equivalent values of $\beta / \beta_{\min }$ for circulation models to those for the outflow models (cf. Table 1). Using these and other measures, it can be shown that the 1D models provide a surprisingly good representation of the $2 \mathrm{D}$ models.

\subsubsection{Model VI: slow disc}

A slowly rotating, thick disc $\left(L \sim 0.6 R^{1 / 2}\right)$, that is very weakly bound $(B \sim-0.02 / R)$, but with similar mass loss index to that of model I. Again $\beta \sim 10$ is large.

\section{TWO-DIMENSIONAL DISCS}

\subsection{Høiland criteria}

In order to make a two-dimensional model of a slowly accreting and consequently hydrostatic disc, we must specify some relationship among the thermodynamical variables $P, \bar{\rho}, S$, etc. Our choice depends upon considerations of stability. An adiabatic, fluid accretion disc naturally develops a negative, radial entropy gradient as heat is generated in its interior. If rotation were unimportant, it would become unstable according to the Schwarzschild criterion. However, a Keplerian disc has a positive angular momentum gradient and, if we were to ignore entropy, it would be stable according to the Rayleigh criterion. For thin discs, the rotational stabilization dominates the entropy destabilization. However, for thick discs, the two effects must be compared directly.

To do this, consider a small, flat ribbon of fluid with an azimuthal length much greater than its width, which, in turn, is much greater than its thickness. Let the ribbon undergo a displacement in the poloidal plane, parallel to its width. Assume that the motion is sufficiently slow that the ribbon remains in pressure equilibrium with its surroundings and sufficiently rapid that its entropy $S$ is unchanged (e.g. Goldreich \& Schubert 1967; Tassoul 1978; Begelman $\&$ Meier 1982). The net buoyant acceleration on the ribbon is then given by

$$
\begin{aligned}
\delta \boldsymbol{a}_{\text {buoy }} & =-(\delta \boldsymbol{r} \cdot \nabla) S\left(\frac{\partial \ln \bar{\rho}}{\partial S}\right)_{\mathrm{P}}\left(\frac{\nabla P}{\bar{\rho}}\right) \\
& =(\delta \boldsymbol{r} \cdot \nabla) S \nabla\left(\frac{P^{1-1 / \gamma}}{1-1 / \gamma}\right),
\end{aligned}
$$


where the spatial gradients are in the surrounding medium. If the displacement is also rapid enough for the specific angular momentum $L$ of the ribbon to be unchanged during the displacement, the surplus centrifugal acceleration of the ribbon relative to its surroundings is likewise given by

$\delta \boldsymbol{a}_{\text {cent }}=-(\delta \boldsymbol{r} \cdot \nabla) L^{2}\left(\frac{\boldsymbol{R}}{R^{4}}\right)=(\delta \boldsymbol{r} \cdot \nabla) L^{2} \nabla\left(\frac{1}{2 R^{2}}\right)$.

The total acceleration is the sum of equations (19) and (20).

If a virtual displacement $\delta \boldsymbol{r}$ is made, the virtual work performed, per unit mass of fluid, is $\delta W=U_{i j} \delta r_{i} \delta r_{j} / 2$, where the tensor $\boldsymbol{U}$ is given by

$\boldsymbol{U}=\nabla\left(\frac{P^{1-1 / \gamma}}{1-1 / \gamma}\right) \otimes \nabla S+\nabla\left(\frac{1}{2 R^{2}}\right) \otimes \nabla L^{2}$.

Only the symmetric part of $\boldsymbol{U}$ need be retained and we can rotate axes in the $r-\theta$ plane so that it is diagonal. The flow will be unstable if there exists a displacement $\delta \boldsymbol{r}$ such that $\delta W>0$. If the trace of $\boldsymbol{U}$ is positive, then there must be unstable displacements. This leads to the first Høiland instability condition,

$\nabla\left(\frac{P^{1-1 / \gamma}}{1-1 / \gamma}\right) \cdot \nabla S+\nabla\left(\frac{1}{2 R^{2}}\right) \cdot \nabla L^{2}>0$.

Discs that satisfy this inequality are unstable in the equatorial plane to radial displacements. If we adopt our self-similar scalings and impose hydrostatic balance in the equatorial plane, then this instability condition can be re-written as

$R^{3} \Omega^{2}<\frac{3 \gamma-5-2 n \gamma+2 n}{\gamma-5-2 n \gamma+2 n} \equiv a(\gamma, n)$

For $\gamma=5 / 3(4 / 3), a(\gamma, n)$ increases from $0(3 / 11)$ to $2 / 7(5 / 13)$ as $n$ increases from 0 to 1 . It turns out that all of the 1 - and twodimensional models discussed below strongly violate inequality (23). For this reason, we argue that adiabatic, fluid discs are \{quite stable to radial convection.

None the less, when $\operatorname{Tr}(\boldsymbol{U})<0$, a second Høiland criterion must be considered and there will still be a range of unstable displacement directions if

$$
\left[\nabla\left(\frac{P^{1-1 / \gamma}}{1-1 / \gamma}\right) \times \nabla\left(\frac{1}{2 R^{2}}\right)\right] \cdot\left[\nabla S \times \nabla L^{2}\right]<0
$$

or

$(\nabla P \times \nabla R) \cdot(\nabla S \times \nabla L)>0$.

In our application, the onset of instability is always associated with a change in sign of $\nabla S \times \nabla L$, not $\nabla P \times \nabla R$.

In order to interpret inequality (25), it is useful to define a series of $2 \mathrm{D}$ surfaces that are tangent on an equatorial ring lying in the disc mid-plane and on which $S, B, L, R, \Omega, r, P, \bar{\rho}$ are constant (Fig. 2). It is straightforward to show, by combining the Høiland criteria with the equation of hydrostatic equilibrium and its curl, that the surfaces of constant $S, B, L, R, \Omega, r, P, \bar{\rho}$, must be nested in order of increasing Gaussian curvature in a stable, fluid disc (Begelman \& Meier 1982; Blandford et al. 1985). [Actually, it is not formally required that the 'isorotes' ( $\Omega=$ constant surfaces) be less curved than spheres, $r=$ constant, though this is true for all of our solutions.]

The surfaces on which the Bernouilli function $B$ is constant are particularly important. We call these 'isoberns'. Using the equation of hydrostatic equilibrium, it can be shown that

$\nabla B=H \nabla \ln S+\Omega \nabla L$.

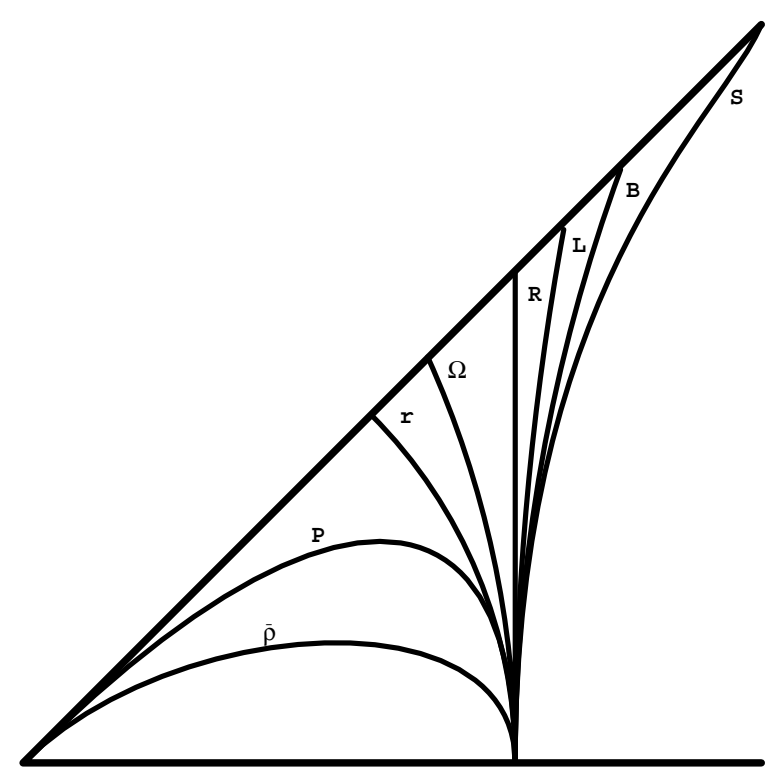

Figure 2. Structure of convectively stable accretion discs. Representative level surfaces of density $(\bar{\rho})$, pressure $(P)$, spherical radius $(r)$, angular frequency $(\Omega)$, cylindrical radius $(R)$, angular momentum $(L)$, Bernouilli function $(B)$ and entropy $(S)$, all passing through the equator at a single radius, are shown in the meridional plane. The horizontal line represents the disc equator and the diagonal line the disc surface. The configuration shown is dynamically stable according to both Høiland criteria equation (25). When the order of the isentropes and isogyres is reversed, the flow becomes convectively unstable.

Equation (26) implies that the isoberns lie between the 'isogyres' (surfaces of constant $L$ ) and the isentropes. Stability requires that the isogyres lie inside the isentropes (equation 25). When the disc is marginally stable to the second Høiland criterion, $S, L, B$ are constant on a common surface, which we call a 'gyrentrope'. We argue that adiabatic, fluid discs should evolve quickly towards this state and we call such discs 'gyrentropic' (cf. Bardeen 1973). In a disc that is marginally unstable to axisymmetric perturbations, the growing eigenmodes have displacements that lie between the isentropes and the isogyres. In other words, near marginal stability, thin ribbons of gas move in opposite directions roughly along the nearly coincident isentrope/isogyre/isobern $=$ gyrentropic surfaces.

Note that we have only considered stability with respect to axisymmetric perturbations. When non-axisymmetric modes are considered the Høiland criteria are necessary rather than sufficient conditions for stability. In particular, baroclinic discs (i.e. discs in which $\nabla P \times \nabla \bar{\rho} \neq 0$ ) can be susceptible to non-axisymmetric modes even when the second Høiland criterion is satisfied (see, e.g., Klahr \& Bodenheimer 2003). For our purposes of demonstrating the convective origin of circulation and outflows, and interpreting $2 \mathrm{D}$ numerical simulations, an axisymmetric treatment suffices.

\subsection{Numerical simulations}

There have been many simulations of fluid dynamical accretion discs, both two- and three-dimensional (3D). In particular, (Stone, Pringle \& Begelman (1999, hereafter SPB99) have carried out twodimensional simulations of adiabatic discs evolving under a variety of prescriptions. Gas was supplied at an intermediate radius and endowed with viscosity. It spread inward (and outward) on a viscous time-scale and became strongly convective, developing a 
gyrentropic structure at intermediate radii. Convection transported energy and angular momentum primarily along gyrentropes. In directions normal to the gyrentropes, the only means of transporting energy and angular momentum were via the viscous stress and advection. Since viscosity transports angular momentum outward with a positive divergence in the equatorial regions, the loss of angular momentum was balanced by a combination of meridional flow and convective transport of angular momentum between high latitudes and the equatorial region. When the gas was marginally unstable according to criterion equation (25), convection exchanged mass, energy and angular momentum between the disc interior and high latitude and not radially outward throughout the disc.

The details of this balance between convection and circulation in the simulations depended upon the form of the viscous stress. For example, when the viscosity $v$ was set to be proportional to $\bar{\rho}$ (run B of SPB99), the viscous torque was balanced partially by inflow in the equatorial zones. However, at high latitudes, where the gyrentropic surfaces themselves became roughly radial, the angular momentum flux was dominated by convective transport and the mass flux was generally outward, producing a quadrupolar flow pattern. When the viscosity was chosen to satisfy the self-similar scaling law, $v \propto r^{1 / 2}$, (run K of SPB99), convection along gyrentropes was strikingly demonstrated in the cross-correlation of the entropy and the angular momentum (Fig. 3).

SPB99 measured separately the radial mass inflow and outflow rates and found that they both obey $\dot{M} \propto r$ very roughly. The net mass accretion rate, given by the inflow rate minus the outflow rate, is approximately independent of $r$ and equals the inflow at the inner

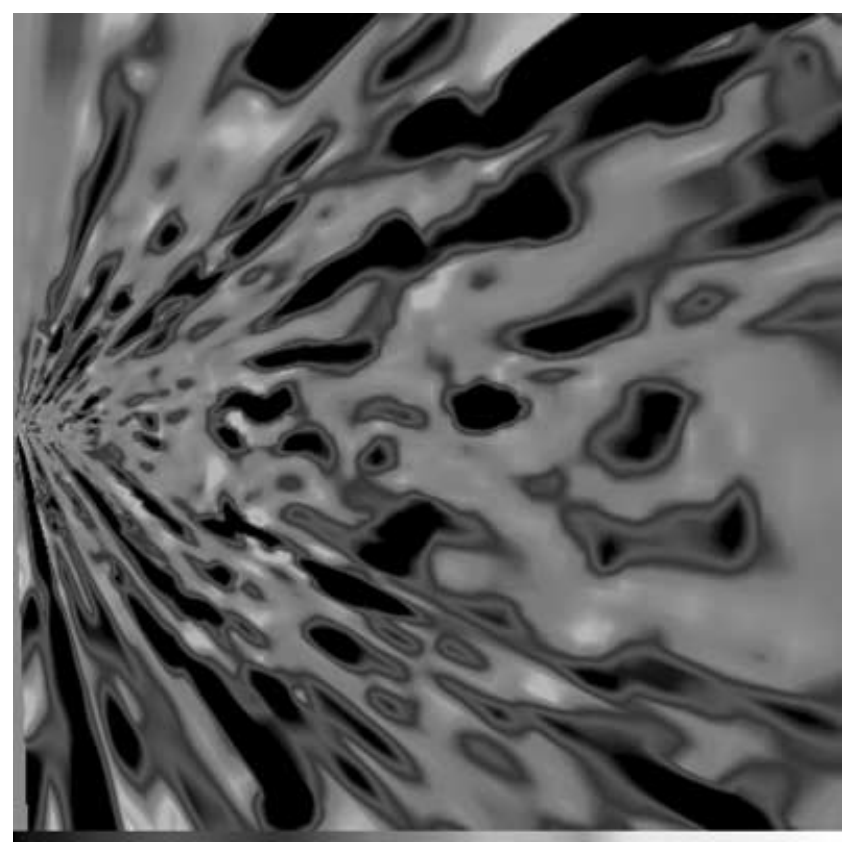

Figure 3. Cross-correlation of specific angular momentum and specific entropy from run $\mathrm{K}$ of SPB99, showing convection along axisymmetric surfaces, which coincide almost exactly with the gyrentropic surfaces. A time-sequence of frames from this simulation shows both inflow and outflow of fluid elements parallel to these surfaces. This is particularly apparent at mid- and high latitudes. The region shown is a meridional slice with the equator bisecting the image horizontally and the black hole and rotation axis off the image toward the left. The radial and vertical extent shown correspond roughly to the inner 10 per cent of the grid, similar to the region shown in fig. 9 of SPB. radius. In other words, the mass reaching the hole at $r_{\text {in }}$ is a fraction $\sim\left(r_{\text {in }} / r_{\text {out }}\right)$ of that supplied at $r_{\text {out }}$. This is a strong indication that the flows had not become stationary, which would probably require a continuous supply of gas at large radius.

\subsection{Global consequences of the gyrentropic hypothesis}

We have argued, on the basis of the one-dimensional treatment, that a rotating flow will have $B>0$ unless there is some means of removing energy. We have also argued that such a flow will become convective and that the natural direction of energy transport is along gyrentropes, where $B$ is constant, to the disc surface. This strongly suggests that adiabatic, fluid discs lose energy though some form of outflow from their surfaces.

However, as emphasized by Abramowicz, Lasota \& Igumenshchev (2000), the condition that $B>0$ somewhere does not automatically imply outflow. Consider a general, axisymmetric disc with surface described by the equation $r=r_{d}(\theta)$. As with a star, the surface properties of a disc can be quite subtle, but it is reasonable to suppose that the enthalpy at the top of the convection zone is much smaller than in the interior and can be ignored. In addition, the pressure will be small; consequently, the surface will be an isobar. Resolving the equation of motion along the disc surface, we obtain a differential equation that relates its shape to the variation of $B$ on the surface (and, indirectly, within the disc):

$\frac{\mathrm{d} \ln r_{d}}{\mathrm{~d} \ln \sin \theta}=-\frac{2(1+B r)}{1+2 B r}$.

From the form of this equation, we discover that the disc surface can have $B>0$ provided that $\mathrm{d} \ln \sin \theta / \mathrm{d} \ln r_{d}<-1 / 2$, that is to say within a funnel where the cross-section increases with height slower than parabolically. However, outside such a funnel, the surface must have $B<0$ if it is to remain bound. Indeed, when the disc latitude decreases with $r$, as it must eventually, then $-1<B r<-1 / 2$ must be satisfied along the free surface. Note, especially, that if the disc surface is conical, as it is in our similarity solutions, then

$B\left(r, \theta_{d}\right)=-\frac{1}{2 r}$

over the entire surface.

The requirement that $B<0$ on the surface of a stable disc (excluding a funnel), however, is inconsistent with having $B>0$ in the interior. This is because the second Høiland criterion, equation (25), requires that $B$ increase with altitude. It is for these reasons that we argue that conservative, adiabatic, fluid accretion discs do not exist and, instead, there must always be outflows.

\subsection{Self-similar, gyrentropic discs}

We now present an elementary description of two-dimensional, selfsimilar, adiabatic, gyrentropic fluid discs. We shall make two key approximations in this description - that the only motion is rotational and that the discs are gyrentropic all the way to their surfaces where the density and pressure vanish simultaneously. Both of these approximations become accurate in the limit that the rate of dissipation tends to zero. In Section 4, we shall introduce a prescription for relaxing the first of these and in Section 5, we shall address the second.

In two dimensions, our assumption of self-similarity requires that the pressure, density and specific angular momentum satisfy the scalings:

$P=r^{n-5 / 2} p(\theta), \bar{\rho}=r^{n-3 / 2} \rho(\theta), L=r^{1 / 2} \ell(\theta)$. 
(Note that we consistently use upper case for the physical variables and lower case for their angular variations.)

The equations of motion are

$\frac{1}{\bar{\rho}} \frac{\partial P}{\partial r}=-\frac{1}{r^{2}}+\frac{L^{2} \csc ^{2} \theta}{r^{3}}$

$\frac{1}{\bar{\rho} r} \frac{\partial P}{\partial \theta}=\frac{L^{2} \cot \theta \csc ^{2} \theta}{r^{3}}$,

from which we obtain

$(5 / 2-n) p / \rho=1-\ell^{2} \csc ^{2} \theta$

$p^{\prime} / \rho=\ell^{2} \cot \theta \csc ^{2} \theta$,

where the prime denotes differentiation with respect to $\theta$.

Now consider the entropy function

$S=P^{1 / \gamma} \bar{\rho}^{-1}=s(\theta) r^{-a /(1-a)}$,

where $a(\gamma, n)$ is defined in equation (23) and $s=p^{1 / \gamma} \rho^{-1}$.

Gyrentropicity implies that $S=S(L)$ and so self-similarity requires that

$S \propto\left(r^{1 / 2} \ell\right)^{-2 a /(1-a)}$.

This provides an algebraic relation among $p, \rho$ and $\ell$.

The solution to equations (32), (33) and (35) is

$$
\begin{aligned}
& \ell=\left\{a+\left[a^{2}+\ell_{0}^{2}\left(\ell_{0}^{2}-2 a\right) \csc ^{2} \theta\right]^{1 / 2}\right\}^{1 / 2} \sin \theta \\
& p=\left(\frac{1-\ell^{2} \csc ^{2} \theta}{5 / 2-n}\right)^{\gamma /(\gamma-1)} s^{-\gamma /(\gamma-1)} \\
& \rho=\left(\frac{1-\ell^{2} \csc ^{2} \theta}{5 / 2-n}\right)^{1 /(\gamma-1)} s^{-\gamma /(\gamma-1)} \\
& s=s_{0}\left(\frac{\ell}{\ell_{0}}\right)^{-2 a /(1-a)},
\end{aligned}
$$

where $\ell_{0}=\ell(\pi / 2)$, etc, measures the angular momentum, etc, in the mid-plane at radius $r_{0}$. Equivalently, we can write

$S=s_{0}\left(\frac{L}{\ell_{0}}\right)^{-2 a /(1-a)}$.

The disc terminates at a free surface where $p, \rho \rightarrow 0$ and so the disc opening angle is given by

$\sin \theta_{d}=l_{d}=\ell_{0}\left(\frac{\ell_{0}^{2}-2 a}{1-2 a}\right)^{1 / 2}$.

We next use equation (36) to solve for the gyrentropes, $L=$ constant These are given by

$r=r_{L}(\theta)=r_{0} \ell_{0}^{2} / \ell^{2}$.

The gyrentropes intersect the disc surface at a radius

$r=r_{d}=r_{0}(1-2 a) /\left(\ell_{0}^{2}-2 a\right)$.

The Bernouilli function, which is also constant on gyrentropes, can be calculated using equation (1) as

$b=r B=-\frac{\ell_{d}^{2}}{2 \ell^{2}}$,

consistent with equation (40). Equation (44) also allows us to relate the value of the Bernouilli function at the mid-plane $(\theta=\pi / 2)$ to the mid-plane angular momentum:

$b_{0}=-\frac{\ell_{0}^{2}-2 a}{2(1-2 a)}$.
Equivalent to equation (40), we have

$B=b_{0}\left(\frac{L}{\ell_{0}}\right)^{-2}$.

The two autonomous relations, equations (40) and (46), are equivalent to the self-similar relation equation (3) and the assumption of gyrentropicity and can be used to replace self-similarity when making a model of the inner disc (cf. Section 6.2). Note that we are only free to specify two functional relations among $L, S, B$ because these relations must be consistent with the equations of motion, equations (30) and (31).

\subsection{Two-dimensional gyrentropic disc models}

For an assumed value of the specific heat ratio, $\gamma$, the above relations suffice to specify a two-parameter family of self-similar disc models. To facilitate comparison with the one-dimensional models, we choose these two additional parameters to be $R^{-1 / 2} L \rightarrow \ell_{0}$ and $R B \rightarrow b_{0}$. The mass loss exponent $n$ can then be thought of as being given implicitly by equation (45), which is identical to the $1 \mathrm{D}$ relation, equation (17). In addition, we must also specify the mid-plane entropy, $s_{0}$, which fixes the pressure and density. However, $s_{0}$ does not change the shape of either the disc or the isogyres and can be set to unity without loss of generality.

The six two-dimensional models, corresponding to the six illustrative one-dimensional models (Section 2.2), are exhibited in Fig. 4. These models demonstrate that the gyrentropes are generically negatively curved surfaces, implying that the isorotes (surfaces of constant $\Omega$ ) are positively curved. They also show that the discs thicken, for a given equatorial angular momentum $\ell_{0}$, as the massloss rate, measured by $n$, decreases. Conversely, for a given value of $n$, the discs thicken as the rotational support, measured by $\ell_{0}$, decreases and the pressure becomes more important. Observe that changing the specific heat ratio is relatively unimportant. In this way, adiabatic accretion is rather different from spherical accretion, where $\gamma=5 / 3$ constitutes a singular limit.

\section{CIRCULATION DISCS}

\subsection{Energy and angular momentum transport}

We have so far treated the disc as being in circular motion and strictly gyrentropic. We now relax the first of these assumptions. Suppose that there is a local torque per unit length

$G \equiv \frac{\mathrm{d} \dot{M} \mathcal{G}}{\mathrm{d} s}$

(where $\mathrm{d} s$ is an element of length in the meridional plane), that is small enough that we can treat its effect as perturbative upon the fundamental, gyrentropic flow pattern. (Note that $G$ is integrated over azimuth.) We conjecture that this torque will eventually drive a fluid disc to marginal instability everywhere, so that the isentropes lie just 'inside' the isogyres and that, except near the disc surfaces, we can continue to approximate the disc angular momentum distribution as gyrentropic.

As was discussed in Section 3.1, the convective motions consist of slender ribbons of fluid moving between these two surfaces. We suppose that the angle between the isogyres and isentropes will open up just enough to allow the relevant transport to take place. Heat and angular momentum can be convected in this manner as a displaced ribbon can exchange both quantities with its surroundings. The relative efficiency with which this happens will depend upon the 

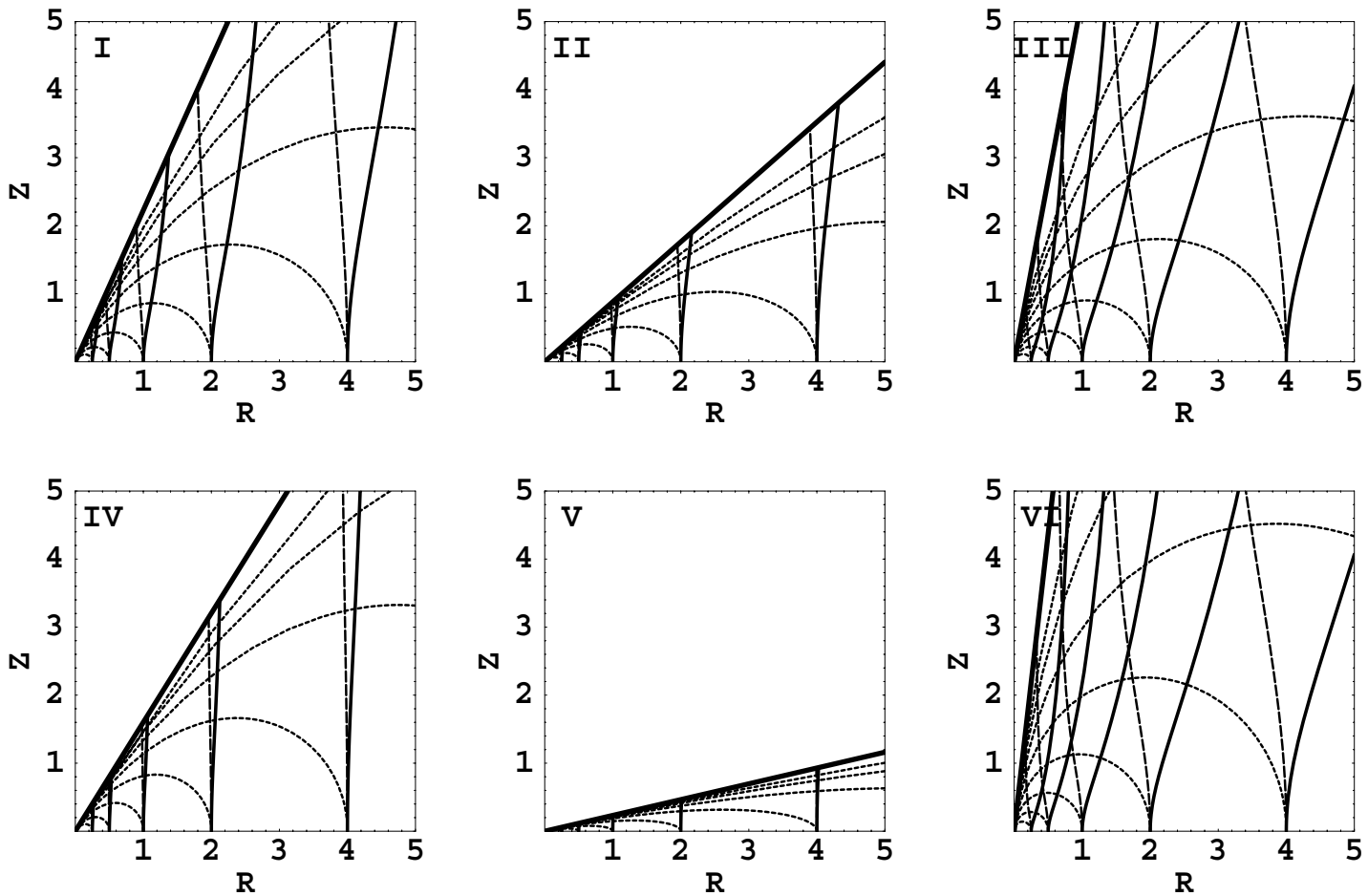

Figure 4. The six simple 2D disc models described in Section 3.5 and Table 1. The isobars, isorotes and gyrentropes are shown with dotted, dashed and solid contours, respectively. In these models, it is assumed that convection is efficient and extends all the way to the disc surface. The outflow does not influence the disc structure in these models.

effective Prandtl number, $P r$, and the presence of alternative transport mechanisms. In our models we assume, without justification, that the thermal conductivity is much lower than the viscosity, $(\operatorname{Pr} \gg$ 1) so that the Newtonian torque $(G \propto-\nabla \Omega)$ will dominate the convective transport of angular momentum, while convective heat transport $(Q)$ will dominate thermal conduction down the temperature gradient. (Alternative prescriptions are certainly possible and we shall consider some in the following paper. Our purpose here is to show how one self-consistent choice allows one to solve for the global disc flow.)

Convection, along gyrentropes, involves relatively rapid, forward and backward motions that average to give a net mass flux per unit length parallel to the gyrentropes, after integrating in azimuth. To this must be added the steady inflow (or outflow) of the gas perpendicular to the gyrentropes. The two flows are combined into a single poloidal mass current vector

$\boldsymbol{J}=2 \pi R \bar{\rho} \boldsymbol{V}_{p}=r^{n-1} \boldsymbol{j}(\theta)$,

where $\boldsymbol{V}_{p} \equiv\left[V_{r}, V_{\theta}\right]$.

\subsection{Torque density}

Consistent with the preceding discussion, we write

$$
\begin{aligned}
\boldsymbol{G} & \equiv r^{n-1 / 2} \boldsymbol{g}(\theta) \\
& =-2 \pi \alpha R^{3} P \nabla \ln \Omega \\
& =3 \pi \alpha \sin ^{3} \theta p\left[1,-2 \omega^{\prime} / 3 \omega\right] r^{n-1 / 2},
\end{aligned}
$$

where $\omega=r^{3 / 2} \Omega=\ell \csc ^{2} \theta$ is the scaled angular velocity, $\alpha \equiv \nu \bar{\rho} \Omega / P$ is the standard viscosity parameter (Shakura \& Sunyaev 1973) and $v$ is the kinematic viscosity. $\boldsymbol{G}$ can be treated as a poloidal vector in this approximation although it is really a third rank tensor. In order to comply with our self-similar assumption, we set $\alpha=$ constant. This ensures that the characteristic inflow and circulation speeds are fixed fractions $(\propto \alpha)$ of the Keplerian value.

\subsection{Conservation laws}

We can now write down equations representing the conservation of mass and angular momentum:

$\nabla \cdot \boldsymbol{J}=0 ; \quad \nabla \cdot(L \boldsymbol{J}+\boldsymbol{G})=0$.

We assume that the torque and poloidal circulation operate on a time-scale much longer than the dynamical time, and can be superimposed perturbatively on the self-similar gyrentropic models derived in Section 3.4. This means that $L$ is given by equations (29) and (36) and the torque density is also expressed using state variables from the gyrentropic models.

Adopting our self-similar prescription, these equations combine to give two ordinary differential equations,

$j_{\theta}^{\prime}=\frac{2 n}{\ell}\left[\ell^{\prime} j_{\theta}+\left(n+\frac{1}{2}\right) g_{r}+g_{\theta}^{\prime}\right]=-n j_{r}$.

This can be solved for an assumed value of $n$ subject to the boundary condition $j_{\theta}(\pi / 2)=0$. Note that solutions to this equation automatically conserve mass. If we start by assuming that $\dot{M} \propto r^{n}$, then the net mass flowing across a hemispherical surface, $\dot{M}=-r \int_{\theta_{d}}^{\pi / 2} \mathrm{~d} \theta J_{r}$, automatically satisfies $\mathrm{d} \dot{M} / \mathrm{d} r=-J_{\theta}\left(\theta_{d}\right)$, as required. The solutions for the flow and the torque (Fig. 5) show that there is a net inflow across the gyrentropes and that the total mass inflow at a given radius in a disc of given shape satisfies $\dot{M} \propto s_{0}^{-\gamma /(\gamma-1)} \alpha$. 

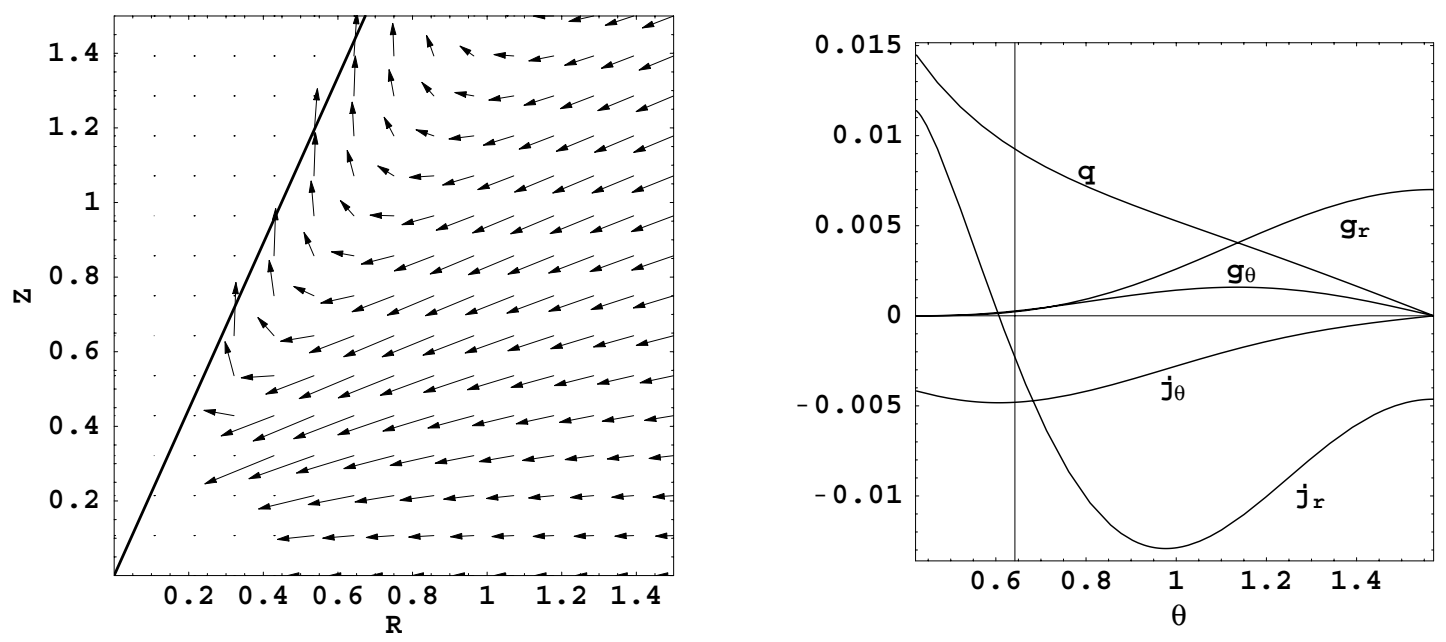

Figure 5. Circulation model for the fiducial illustrative example I. (a) Poloidal mass flux $\boldsymbol{j}$ from equation (53). It is assumed that the mass flow extends all the way to the disc surface. Note that, although the net poloidal flow is inward, the flow is actually outward near the disc surface. (b) The two components of the mass flux $\boldsymbol{j}$ and the assumed viscous torque $\boldsymbol{g}$, and the convective heat flow along the gyrentropes, measured by $q$. The vertical line marks the location of the thermal front in the outflow model (cf. Section 5).

However, superimposed upon this inflow is a quadrupolar circulation, directed inward in the equatorial region and outward near the disc surface. In some, though not all, solutions the combined, radial flow is outward at high latitude.

Although the solution for $\boldsymbol{J}$ is finite at the disc surface, the velocity $\boldsymbol{V}=\boldsymbol{J} / 2 \pi R \bar{\rho}$ diverges. This signals the failure of our model for convective energy transport, as we discuss in the following section. In contrast, the torque density $\boldsymbol{G}$ vanishes at the disc surface according to equation (49). The angular momentum incident on the disc surface is then

$\frac{\mathrm{d} F_{L}}{\mathrm{~d} r}=-L J_{\theta}=L \frac{\mathrm{d} \dot{M}}{\mathrm{~d} r} \Rightarrow \eta=0$,

where we have used equation (5). This confirms that the outflowing gas only carries off its own specific angular momentum, cf. equation (7), BB99.

We next solve for the convective energy flux $\boldsymbol{Q}$. This must be directed along the gyrentropes and so we can write it in the form

$\boldsymbol{Q} \equiv\left[2 \ell^{\prime},-\ell\right] q(\theta) r^{n-2}$.

The equation of energy conservation becomes

$\nabla \cdot[B \boldsymbol{J}+\Omega \boldsymbol{G}+\boldsymbol{Q}]=0$.

This furnishes a first-order differential equation for $q(\theta)$,

$\ell q^{\prime}-(2 n-3) \ell^{\prime} q+j_{r} b-j_{\theta} b^{\prime}-(n-1) \omega g_{r}-\omega^{\prime} g_{\theta}-\omega g_{\theta}^{\prime}=0$,

which can be solved subject to $q(\pi / 2)=0$.

The supply of energy to the disc surface is given by

$\frac{\mathrm{d} F_{E}}{\mathrm{~d} r}=J_{\theta} B+Q_{\theta}$.

Comparing with equation (9) we deduce that

$\beta=\frac{Q_{\theta}}{J_{\theta} B}=\frac{\ell_{d} q_{d}}{j_{\theta d} b_{d}}=\frac{-2 \sin \theta_{d} q_{d}}{j_{\theta d}}$,

where $\ell_{d}, b_{d}$ are given by equations (41) and (28) and $j_{\theta d}, q_{d}$ are given by solutions of the differential equations (53) and (57). $\beta$ is then a function of $\ell_{0}, n$ and, implicitly of $\ell_{0}, b_{0}$ as in the $1 \mathrm{D}$ models.

In the important limit when the disc expands all the way to the pole, with $\theta_{d}, \ell_{d} \rightarrow 0$ and $\ell_{0} \rightarrow(2 a)^{1 / 2}, b_{0} \rightarrow 1 / 2$, then $\beta \rightarrow \infty$.

\subsection{Circulation disc models}

We have computed circulation disc models corresponding to the 1D, 2D models discussed in Sections 2.2 and 3.5. In Fig. 5, we exhibit the flow pattern for our fiducial model I (adopting a viscosity parameter $\alpha=0.03$ ). We also present the angular variations of the mass flux, torque and convective heat flux. The poloidal flow is fundamentally quadrupolar, inward at low latitude and outward at high latitude. The flows for the other five circulation models are qualitatively similar.

\section{OUTFLOW DISCS}

\subsection{Efficiency of convection}

We now turn to the second of our modifications to the basic gyrentropic disc model, which introduces features that may be largely absent from existing numerical simulations due to inadequate resolution and/or missing physical processes. We allow for the fact that convection of energy and angular momentum will not be efficient close to the disc surface so that the gyrentropic approximation may no longer hold. We have already argued that the convection will be along the isogyres. Assuming that the isogyres and the isentropes have the unstable ordering with an angle $\delta$ between them, then, according to standard mixing-length theory (e.g. Hansen \& Kawaler 1994), the convective energy flux, integrated over azimuth, will be given by

$$
\begin{aligned}
Q & \sim 2 \pi R \bar{\rho} v_{\mathrm{c}}^{3} \\
& \sim 2 \pi R P^{3 / 2} \bar{\rho}^{-1 / 2}\left(\boldsymbol{h}_{\mathrm{P}} \cdot \nabla \ln S\right)^{3 / 2} \\
& \sim 2 \pi R P^{3 / 2} \bar{\rho}^{-1 / 2} \delta^{3 / 2},
\end{aligned}
$$

where $v_{\mathrm{c}}$ is the convection speed and $\boldsymbol{h}_{\mathrm{P}}$ is a vector of length equal to the pressure scaleheight and tangent to the isogyre. (We drop numerical constants of the order of unity.) The Mach number of the convective motions is given by

$$
M_{c} \sim \delta^{1 / 2} \sim\left(\frac{Q \bar{\rho}^{1 / 2}}{2 \pi R P^{3 / 2}}\right)^{1 / 3} \sim\left(\frac{q \ell \rho^{1 / 2}}{2 \pi p^{3 / 2} \sin \theta}\right)^{1 / 3} .
$$




\subsection{Thermal front}

We now make the Ansatz that, when $M_{c}$ reaches a certain critical value $M_{c, c r i t}$, which we choose, arbitrarily, though not unreasonably, to be unity, the convective motions rapidly dissipate and the convective energy flux is quickly converted into heat, increasing the entropy of the gas. Simultaneously, we suppose that the torque ceases to act and that the viscous transport of angular momentum and energy stops abruptly. In reality, this transition is likely to occur gradually through the dissipation of turbulent wave modes and the acceleration of the flow within an extended region. However, approximating this transition as a discontinuity, located at a biconical thermal front, suffices for us to make a simple model.

We impose jump conditions at this transition by requiring that the mass, momentum and energy fluxes are equal on either side of the transition

$$
\begin{aligned}
& {\left[J_{\theta}\right]=0 \Rightarrow j_{\theta w}=j_{\theta, \mathrm{th}}} \\
& {\left[J_{\theta} V_{r}\right]=0 \Rightarrow v_{r, w}=v_{r, \mathrm{th}}} \\
& {\left[2 \pi R P+J_{\theta} V_{\theta}\right]=0} \\
& \quad \Rightarrow j_{\theta, \mathrm{th}}\left(v_{\theta, w}-v_{\theta, \mathrm{th}}\right)=2 \pi \sin \theta_{\mathrm{w}}\left(p_{\mathrm{th}}-p_{\mathrm{w}}\right) \\
& {\left[G_{\theta}+J_{\theta} L\right]=0 \Rightarrow j_{\theta, \mathrm{th}}\left(\ell_{\mathrm{w}}-\ell_{\mathrm{th}}\right)=g_{\theta, \mathrm{th}}} \\
& {\left[G_{\theta} \Omega+J_{\theta} B+Q_{\theta}\right]=0} \\
& \Rightarrow j_{\theta, \mathrm{th}}\left(b_{\mathrm{th}}-b_{\mathrm{w}}\right)=\ell_{\mathrm{th}} q_{\mathrm{th}}-g_{\theta, \mathrm{th}} \omega_{\mathrm{th}} .
\end{aligned}
$$

These equations allow us to solve for the initial physical conditions (specifically $p_{\mathrm{w}}, \rho_{\mathrm{w}}, \boldsymbol{v}_{\mathrm{w}}$ ) at the base of the wind (designated w) in terms of those upstream from the thermal front (designated th). The disc now terminates at an opening angle $\theta_{\mathrm{w}}$ rather than $\theta_{d}$ and equations (54) and (59) must be replaced with

$\eta=\frac{g_{\theta, \mathrm{th}}}{\ell_{\mathrm{th}} j_{\theta, \mathrm{th}}}$

$\beta=\frac{\ell_{\mathrm{th}} q_{\mathrm{th}}-g_{\theta, \mathrm{th}} \omega_{\mathrm{th}}}{j_{\theta, \mathrm{th}} b_{\mathrm{th}}}$.

Note that $\eta$ will now be negative because the isorotes are less curved than spheres. This is an artificial consequence of locating the effective disc surface inside the disc. In general, $\eta$ is usually small for fluid discs and it is a fair approximation to ignore it.

\subsection{Wind solutions}

We now turn to the structure of a thermally driven, gas dynamical wind and describe simple similarity solutions along the lines of those first derived by Bardeen \& Berger (1978). We suppose that a gas dynamical wind is launched from the surface of the disc located at $\theta=\theta_{\mathrm{w}}$ with initial conditions determined by the jump conditions at the thermal front. In the solutions that we shall consider, the increase in entropy at the thermal front is sufficient to change the sign of the Bernouilli function and render the gas unbound. We suppose that in its subsequent flow we can ignore viscous stress and mixing of mass, angular momentum and energy between streamlines.

We continue to adopt the self-similar scalings and generalize the velocity to a three-dimensional vector,

$\boldsymbol{V}=r^{-1 / 2}\left[v_{r}(\theta), v_{\theta}(\theta), v_{\phi}(\theta)\right]$, in spherical polar coordinates. The equations of continuity, motion and entropy conservation can be written as

$\frac{\rho^{\prime}}{\rho}+\frac{v_{\theta}^{\prime}}{v_{\theta}}=-\cot \theta-\frac{n v_{r}}{v_{\theta}}$

$\frac{v_{r}^{\prime}}{v_{r}}=\frac{\left(v_{r}^{2}+2 v_{\theta}^{2}+2 v_{\phi}^{2}-2\right) \rho+(5-2 n) p}{2 \rho v_{r} v_{\theta}}$

$\frac{v_{\theta}^{\prime}}{v_{\theta}}+\left(\frac{p}{\rho v_{\theta}^{2}}\right) \frac{p^{\prime}}{p}=\frac{2 v_{\phi}^{2} \cot \theta-v_{r} v_{\theta}}{2 v_{\theta}^{2}}$

$\frac{v_{\phi}^{\prime}}{v_{\phi}}=-\frac{v_{r}}{2 v_{\theta}}-\cot \theta$

$\frac{1}{\gamma} \frac{p^{\prime}}{p}-\frac{\rho^{\prime}}{\rho}=\left(\frac{a}{1-a}\right) \frac{v_{r}}{v_{\theta}}$,

where a prime denotes differentiation with respect to $\theta$.

These equations can now be rearranged:

$$
\begin{aligned}
p^{\prime}= & {\left[\gamma p \rho \left\{[1+2 n(a-1)+a] v_{r} v_{\theta}\right.\right.} \\
& \left.\left.+2(a-1)\left(v_{\phi}^{2}+v_{\theta}^{2}\right) \cot \theta\right\}\right]\left[2(a-1)\left(\gamma p-\rho v_{\theta}^{2}\right)\right]^{-1} \\
\rho^{\prime}= & -\left[\rho \left\{v_{r}\left[2 \gamma p a+(2 n-1)(a-1) \rho v_{\theta}^{2}\right]\right.\right. \\
& \left.\left.+2(a-1) \rho v_{\theta}\left(v_{\phi}^{2}+v_{\theta}^{2}\right) \cot \theta\right\}\right] \\
& \times\left[2(1-a) v_{\theta}\left(\gamma p-\rho v_{\theta}^{2}\right)\right]^{-1} \\
v_{r}^{\prime}= & \frac{(5-2 n) p+\rho\left(2 v_{\phi}^{2}+v_{r}^{2}+2 v_{\theta}^{2}-2\right)}{2 \rho v_{\theta}} \\
v_{\theta}^{\prime}= & \left\{v_{r}\left[-2 \gamma p(a+n a-n)+(a-1) \rho v_{\theta}^{2}\right]\right. \\
& \left.-2(a-1)\left(\gamma p+\rho v_{\phi}^{2}\right) v_{\theta} \cot \theta\right\} \\
& \times\left[2(a-1)\left(\gamma p-\rho v_{\theta}^{2}\right)\right]^{-1} \\
v_{\phi}^{\prime}= & -\frac{v_{\phi}\left(v_{r}+2 v_{\theta} \cot \theta\right)}{2 v_{\theta}} .
\end{aligned}
$$

These equations have three integrals, as may be readily verified. The entropy $S$, the Bernouilli function $B$ and the specific angular momentum $L$ are conserved along streamlines. We conclude that, as with the disc, $S=S(L), B=B(L)$ in the wind, although for a quite different reason. The entire flow can then be labelled using the disc gyrentropes that extend out to the thermal front where they are replaced by the wind gyrentropes.

Now consider the wind flow well away from the disc. There is potentially a critical point where $v_{\theta}=(\gamma p / \rho)^{1 / 2}$, the adiabatic speed of sound. (The reason why only the $\theta$ component of the velocity is involved is that self-similarity and axisymmetry prescribe the other two components.) However, solutions satisfying appropriate boundary conditions never reach their critical speeds. From a mathematical point of view, these winds are 'breezes', and $v_{\theta}$ is always subsonic, even if the radial velocity becomes strongly supersonic. (In fact, $v_{\theta}$ must be subsonic, under all conditions, for $\gamma<3 / 2$.)

As $B$ is conserved in the outflow we can use its value at the thermal front to compute the asymptotic wind speed. We express the speed, $v_{\infty}$, by tracing the flow back to the thermal front on a flow line and then back to the mid-plane on a gyrentrope. $v_{\infty}$ is then given in Table 1 in units of the Keplerian speed at this point.

\subsection{Outflow disc models}

We have integrated equations (77)-(81) and produced matched flows for the six examples (Fig. 6). We find that it is generally possible 

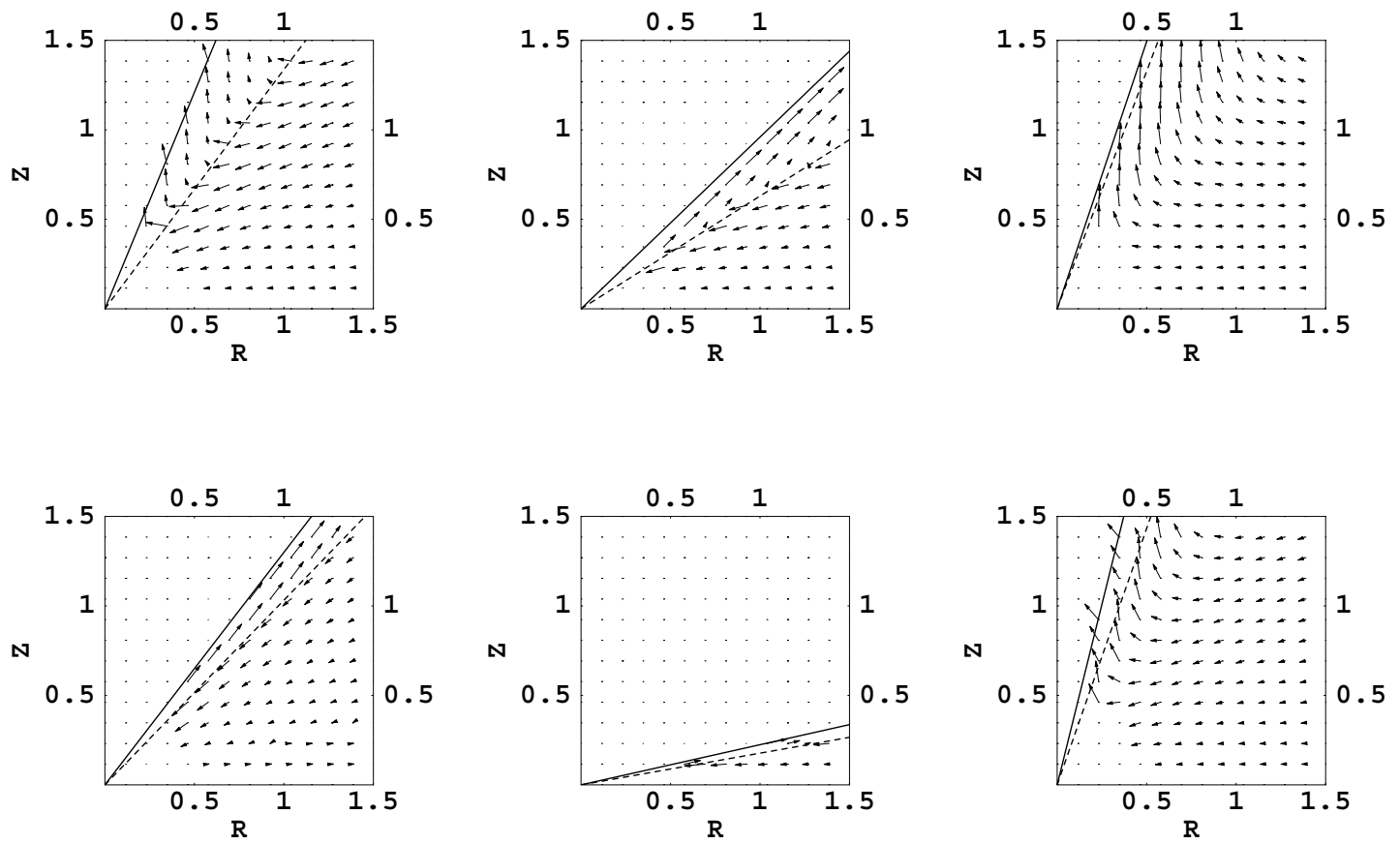

Figure 6. Poloidal flows for the six outflow disc models listed in Section 2.2 and Table 1. Within the disc, the conditions are identical to those presented in Fig. 5. However, the disc is presumed to terminate at a thermal front (with opening angle $\theta_{\mathrm{w}}$; dashed line) where the convective heat flux and the viscosity abruptly vanish and across which the flows of mass, momentum and energy are conserved. This is where the outflow begins (cf. Section 5.2). The outflow lies between cones with opening angles $\theta_{j}, \theta_{\mathrm{w}}$. The flow at small radius is omitted.

to obtain physically plausible solutions for viable disc models. For each of our examples, which span a much larger volume of parameter space than we envisage is occupied by real discs, we find that the viscous dissipation creates so much heat that it easily unbinds the gas downwind of the thermal front. The outflow always occupies a hollow cone on account of the centrifugal barrier. The computed thermal fronts lie somewhat below the surfaces of the computed 2D discs (i.e. $\theta_{\mathrm{w}}>\theta_{d}$ ) and so the outflow discs are somewhat thinner than the equivalent circulation discs, but not by a large factor for reasonable values of $\alpha$ and $M_{c \text {,crit }}$. The jet cones have angles $\theta_{j}$ that are not much smaller than the thermal front cone angles, $\theta_{\mathrm{w}}$. The values of $\beta$ computed in these more complete models do not differ greatly from those in our simple 1D and circulation disc models. The values of the angular momentum loss parameter $\eta$ are generally small except for model IV, where it is compensating for the low mass-loss rate.

We have made a very simple, two-part model to account for the dissipation. We suppose that there is distributed, Newtonian dissipation within the disc, and a discontinuous entropy production front at the disc surface. How do our results depend upon the choice of $\alpha$, which controls the former process, and $M_{c, \text { crit }}$, which controls the latter? For model I, we find that increasing $\alpha$ from 0.03 to 0.1 causes the thermal front to be located at greater density in the disc, where the opening angle is $\theta_{\mathrm{w}}=0.78$ as opposed to 0.64 . The cone excluded by the jet has a correspondingly larger opening angle, increasing to $\theta_{j}=0.46$ from 0.39 . Conversely, when we reduce $\alpha$ to 0.01 , the thermal front is located at $\theta_{\mathrm{w}}=0.57$, i.e. closer to the surface of the original gyrentropic disc at $\theta_{d}=0.42$, so that the original gyrentropic model becomes more accurate. (In addition, $\theta_{j}$ falls to 0.33.)

When we separately reduce $M_{c \text {,crit }}$ to 0.7 we find that the thermal front is located deeper in the disc, at $\theta_{\mathrm{w}}=0.76$, and that the jet cone angle increases to $\theta_{j}=0.55$ as the outflow is launched with a lower speed relative to the surface of the thermal front. Our models are therefore not strongly sensitive to the values of $\alpha$ and $M_{c, \text { crit }}$, and the sense of the changes that variations in these parameters bring about are as expected.

\section{NON-SELF-SIMILAR FLOW AT SMALL AND LARGE RADII}

\subsection{Energy release in adiabatic flows}

We have so far concentrated upon self-similar models of adiabatic accretion flows because these allow us to generate self-consistent solutions through solving ordinary differential equations. However, as was made clear in BB99, the requirement that energy be carried away somehow from an adiabatic flow follows from general conservation laws and is not dependent upon self-similarity. If, for example, the viscous torque were to decrease suddenly at the radius where the disc becomes neutral, the thickness and the outflow would also be expected to exhibit abrupt changes. However, there would still be a need for the energy released at small radius to be carried off in an outflow. In the context of the present paper, the discs should still convect energy to their surfaces and drive meridional circulation.

Furthermore, self-similar solutions cannot describe important features of the flow at small and large radius where self-similarity must fail. In this section, we discuss relativistic flow at small radius and non-self-similar flow near the outer transition radius.

\subsection{Relativistic flow at small radius}

A convenient form of the equation of hydrostatic balance for a stationary, axisymmetric flow is

$$
-\frac{\nabla P}{\tilde{\rho} \tilde{H}}=\overrightarrow{\tilde{A}}=\nabla \ln \tilde{E}-\frac{\tilde{\Omega} \nabla \tilde{L}}{1-\tilde{\Omega} \tilde{L}},
$$



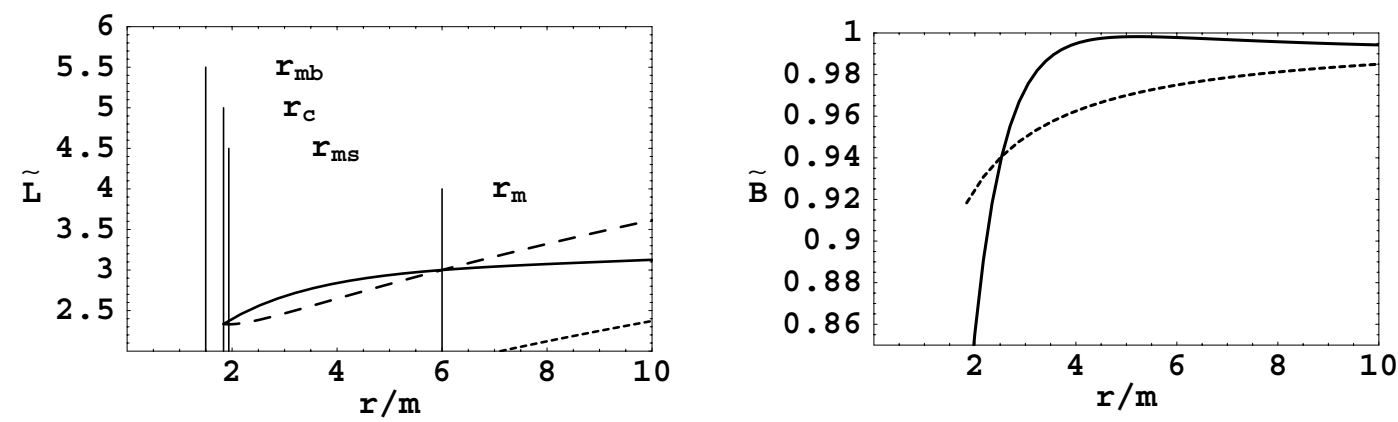

Figure 7. (a) Assumed relativistic, fluid angular momentum variation, $\tilde{L}$ (solid) for a gyrentropic disc around a Kerr hole with $a=0.9 \mathrm{~m}$. The Keplerian variation (dashed) is shown for comparison. The two angular momenta must coincide at the cusp radius $r_{\mathrm{c}}=1.84 \mathrm{~m}$ and at the pressure maximum at $r_{\mathrm{m}}=$ $6 \mathrm{~m}$. At large radius the fluid angular momentum asymptotes to the model I self-similar solution (dotted). Also shown are the radius of zero binding energy $r_{\mathrm{mb}}=1.5 \mathrm{~m}$ and the radius of marginal stability $r_{\mathrm{ms}}=1.93 \mathrm{~m}$. (b) Derived variation of the relativistic Bernouilli function $\tilde{B}\left(r_{0}\right)$ (dashed). Like the entropy, it asymptotes quickly to the non-relativistic form $B\left(r_{0}\right)=b_{0} r_{0}^{-1}$.

where $c=1, \overrightarrow{\tilde{A}}$ is the acceleration and the relativistic enthalpy per unit rest mass is $\tilde{H}=1+H=1+\gamma P /(\gamma-1) \tilde{\rho}$, with $\tilde{\rho}$ now representing the proper density of rest mass. This applies to both radiation-dominated $(\gamma=4 / 3)$ and ion-dominated $(\gamma=5 / 3)$ flows. $\tilde{E}=-u_{0}$ is the energy at infinity, $\tilde{\Omega}=u^{\phi} / u^{0}$ is the angular velocity and $\tilde{L}=-u_{\phi} / u_{0}$ is the fluid angular momentum. [Note that the relativistic definition of angular momentum differs from the conventional dynamical choice of $u_{\phi}$. It is more convenient for fluid dynamical use (Bardeen 1973; Seguin 1975).] These essentially kinematical quantities are related through

$\tilde{E}(r, \theta, \tilde{L})=\left(-g^{00}+2 g^{0 \phi} \tilde{L}-g^{\phi \phi} \tilde{L}^{2}\right)^{-1 / 2}$

$\tilde{\Omega}(r, \theta, \tilde{L})=\frac{g^{0 \phi}-g^{\phi \phi} \tilde{L}}{g^{00}-g^{0 \phi} \tilde{L}}$,

where $g^{\alpha \beta}$ is the contravariant form of the metric tensor in BoyerLindquist coordinates for a hole with specific angular momentum

$a$. For illustration we adopt the value $a=0.95 \mathrm{~m}$.

The relativistic generalization of equation (26) is

$\nabla \ln \tilde{B}=\left(1-\tilde{H}^{-1}\right) \nabla \ln S+\frac{\tilde{\Omega} \nabla \tilde{L}}{1-\tilde{\Omega} \tilde{L}}$,

where

$\tilde{B}=\tilde{H} \tilde{E}$

is the relativistic Bernouilli function and the entropy function is still given by equation (2). Note that the Bernouilli function now includes a contribution from the rest mass.

The two non-relativistic Høiland criteria, equations (22) and (25), have relativistic counterparts (Seguin 1975; Blandford et al. 1985). The first criterion for marginal instability generalizes to become:

$\frac{1}{\tilde{H}^{2}} \nabla\left(\frac{P^{1-1 / \gamma}}{1-1 / \gamma}\right) \cdot \nabla S-\vec{\gamma} \cdot \nabla \tilde{L}>0$,

where

$\vec{\gamma}=\frac{\mathrm{d} \overrightarrow{\tilde{A}}}{\mathrm{~d} \tilde{L}}=\frac{E^{4} \nabla \tilde{L}}{g_{0 \phi}^{2}-g_{00} g_{\phi \phi}}-\frac{\nabla \tilde{\Omega}}{(1-\tilde{\Omega} \tilde{L})^{2}}$

and the derivative is performed assuming only circular motion. The second criterion also generalizes to become

$(\nabla P \times \vec{\gamma}) \cdot(\nabla S \times \nabla \tilde{L})>0$.

We find that the first Høiland criterion is even more strongly satisfied in the inner disc (where the entropy gradient changes to become stabilizing) than in the self-similar disc. We conjecture that the flow will evolve to a state of marginal stability according to the second Høiland criterion so that the entire disc is gyrentropic.

In order to construct a gyrentropic inner disc model, we need to introduce two autonomous functions $S(\tilde{L}), \tilde{B}(\tilde{L})$ as described in Section 3.4. What we actually do is ultimately equivalent. We specify equatorial distributions of angular momentum and entropy, $\tilde{L}\left(r_{0}\right), S\left(r_{0}\right)$, from which we can deduce $S(\tilde{L})$, that are chosen to match on to the non-relativistic functions at large radius. In addition, the angular momentum must equal the relativistic Keplerian value at two radii - a radius $r_{\mathrm{m}}$ where the pressure is maximized and a smaller radius $r_{\mathrm{c}}$ (the 'cusp' radius) where the pressure and its gradient vanish. The particular form of $\tilde{L}$ that we use is

$\tilde{L}\left(r_{0}\right)=\ell_{0} r_{0}^{1 / 2}\left[1+c_{1} \exp \left(-c_{2} r_{0}\right)\right]$,

where the constants $c_{1}=1.78, c_{2}=0.18$ are chosen to fit a disc with $r_{\mathrm{c}}=1.84 \mathrm{~m}, r_{\mathrm{m}}=6 \mathrm{~m}$ (Fig. 7).

The entropy function is required to satisfy the first Høiland criterion and has to be tuned to allow the Bernouilli function, derived below, to match the non-relativistic form $B\left(r_{0}\right)=b_{0} r_{0}^{-1}$ at large radius. We use

$S\left(r_{0}\right)=r_{0}^{-a /(1-a)}\left[1+c_{3} \exp \left(-c_{4} r_{0}\right)\right]$.

The choices $c_{3}=5.0, c_{4}=0.85$ produce a suitable solution.

In order to compute the Bernouilli function, we use equation (83) to compute the energy $\tilde{E}$ and the angular velocity $\tilde{\Omega}$ as functions of equatorial radius $r_{0}$. We then rewrite equation (84) in the form

$\frac{\mathrm{d} \tilde{B}}{\mathrm{~d} r_{0}}=\left[\tilde{B}-\tilde{E}\left(r_{0}\right)\right] \frac{\mathrm{d} \ln S}{\mathrm{~d} r_{0}}\left(r_{0}\right)+\frac{\tilde{\Omega}\left(r_{0}\right) \tilde{B}}{1-\Omega\left(r_{0}\right) \tilde{L}\left(r_{0}\right)} \frac{\mathrm{d} \tilde{L}}{\mathrm{~d} r_{0}}$

and solve this differential equation to infer the variation $\tilde{B}\left(r_{0}\right)$ and, consequently, $\tilde{B}(\tilde{L})$ (Fig. 7).

The next step is to solve for the disc structure away from the equatorial plane. Again we use equation (84), this time written in the form:

$\frac{\mathrm{d} \tilde{B}}{\mathrm{~d} \tilde{L}}(\tilde{L})=[\tilde{B}(\tilde{L})-\tilde{E}(r, \theta, \tilde{L})] \frac{\mathrm{d} \ln S}{\mathrm{~d} \tilde{L}}(\tilde{L})+\frac{\tilde{\Omega}(r, \theta, \tilde{L}) \tilde{B}(\tilde{L})}{1-\Omega(r, \theta, \tilde{L}) \tilde{L}}$

and solve this for $\tilde{L}(r, \theta)$. This enables us to calculate $S(r, \theta), B(r$, $\theta$ ) and then to compute $E(r, \theta), \Omega(r, \theta)$ using equation (83). Finally, we can calculate the pressure using

$P=\left[\left(\frac{\gamma-1}{\gamma S}\right)\left(\frac{\tilde{B}-\tilde{E}}{\tilde{E}}\right)\right]^{\gamma /(\gamma-1)}$

and plot the isobars (Fig. 8). 


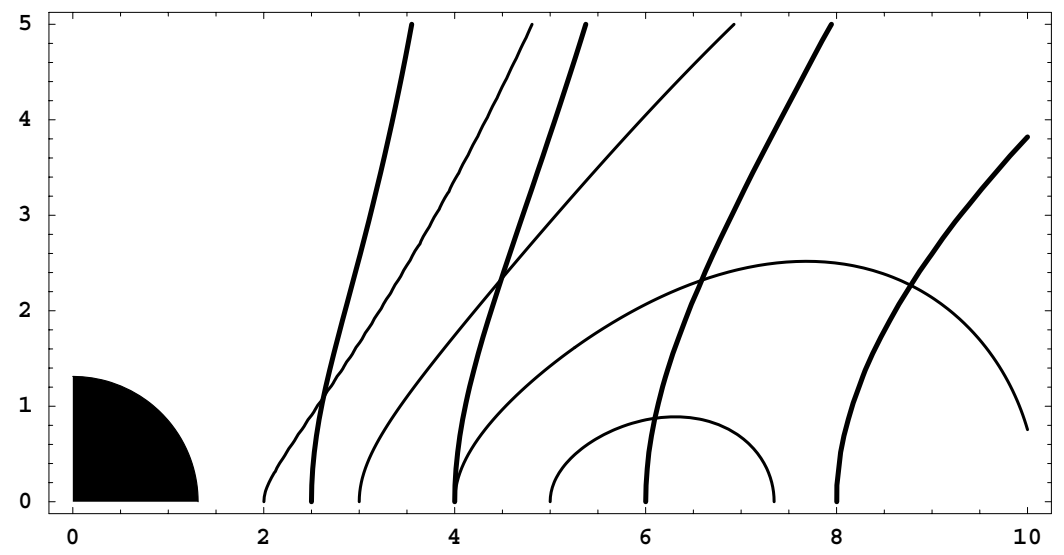

Figure 8. Model of the inner regions of gyrentropic accretion disc orbiting a Kerr black hole with the assumed angular momentum and entropy distributions displayed in Fig. 7. The gyrentropes are displayed as bold contours. The cusp is located between the marginally bound and marginally stable orbits. The isobars are shown as light contours surrounding the pressure maximum.

Although our choice of functions in equations (89) and (90) is arbitrary and has no immediate physical basis, our procedure does illustrate how to construct self-consistent disc models given a more comprehensive theory of relativistic convection. It is straightforward, in principle, to generalize the non-relativistic, self-similar development of circulation and outflow disc models to the nonself-similar regime. We could use the relativistic generalizations of equations (52) and (56) to derive partial differential equations for the poloidal velocity for a non-self-similar circulation and outflow. However, there is little point in carrying out this exercise as the assumed autonomous relations that define the gyrentropes are arbitrarily prescribed. It is, instead, more valuable to solve the timedependent 2D and 3D fluid dynamical equations numerically, making different assumptions concerning the viscosity and, perhaps, the heat transport. The principles that we have developed here should be of use in interpreting such a computation.

\subsection{Transition disc}

Self-similarity must also break down at some outer radius, which either represents an outer boundary condition associated with the mass supply or the radius beyond which radiative losses are significant. In the ion-dominated case, cooling near the outer transition radius $r_{\mathrm{t}}$ (i.e. between a thin radiative disc and an ADIOS) is likely to be dominated by thermal bremsstrahlung of the one-temperature $\left(T_{e}=T_{i}\right.$ ) plasma, provided that $r_{\mathrm{t}}>2000 \mathrm{~m}$ (where $m=G M / c^{2}$ is the gravitational radius). Equating the cooling rate and the inflow time, we find that adiabatic flow is possible for

$r_{\mathrm{t}}<10 \frac{\alpha^{4}}{\dot{m}_{\mathrm{t}}^{2}} m$

where $\dot{m}_{\mathrm{t}} \equiv \dot{M}_{\mathrm{t}} / \dot{M}_{E}$ is the mass flux crossing into the non-radiative region (the mass supply). Given a power-law scaling of $\dot{m}$ with index $n$ at $r<r_{\mathrm{t}}$, the mass flux reaching the black hole (the accretion rate) is

$\dot{m}_{\mathrm{acc}}<10^{-9 n} \alpha^{-4 n} \dot{m}_{\mathrm{t}}^{1+2 n}$.

For example, if $\dot{m}_{\mathrm{t}}=0.01, \alpha=0.03$ and $n=0.6$ (as in model I), the accretion rate can be as low as $\dot{m}_{\text {acc }} \sim 10^{-6}$, four orders of magnitude lower than the supply rate. Since $\dot{m}_{\text {acc }} \ll 50 \alpha^{2} \sim 0.05$, the ADIOS easily satisfies the condition for a two-temperature flow close to the black hole (Rees et al. 1982) - which is more stringent than the condition for adiabatic flow at large radii - implying that the adiabatic flow, once established, will extend all the way from $r_{\mathrm{t}}$ in the vicinity of the hole. For conservative inflow models, this condition is more difficult to satisfy. Moreover, for the parameters we have chosen $r_{\mathrm{t}}$ can be as large as $\sim 10^{7} \mathrm{~m}$; thus the flow could exhibit self-similarity over several decades in radius.

Similar considerations apply to accretion flows with $\dot{m} \gg 1$. Here the transition from radiative to non-radiative flow occurs at the trapping radius, $r_{\mathrm{t}} \sim r_{\text {trap }} \sim \dot{m}_{\mathrm{t}} m$ (Begelman 1979). The accretion rate is given by $\dot{m}_{\mathrm{acc}} \sim \dot{m}_{\mathrm{t}}^{1-n}$.

We note that models for the transition between the thin disc and non-radiative flow have been studied mainly in the context of ADAF models, in which conserved mass flux through the disc is assumed (e.g. Kato \& Nakamura 1998; Manmoto et al. 2000). These models generally require a zone of anomalous emissivity near $r_{\mathrm{t}}$ in order to soak up the outward energy flux, which cannot be accepted by the thin disc in a smooth transition. This problem is avoided if we allow for the onset of mass (and angular momentum) loss in the transition region.

In order to demonstrate that a smooth transition is possible under ADIOS models, we combine the analyses of Sections 2.1 and 6.2 and make a one-dimensional model for a disc where we choose functional forms for the radial variation of $L, B, \mathcal{G}$ that interpolate between the thick and thin limits. We emphasize that these functions, although plausible, have no physical basis and are only intended to demonstrate how a physical solution might be constructed. In particular, these models are not consistent with a simple $\alpha$-type parametrization of the viscous couple, in which $\mathcal{G}$ is an increasing function of pressure. It may not be possible to construct selfconsistent models for all reasonable forms of $\mathcal{G}$; in some cases, solutions may be time-dependent.

We choose to match to model I and adopt a self-similar thin disc with $\ell_{\text {out }}=0.95, b_{\text {out }}=-0.45$. We require that the mass and angular momentum loss rates $\rightarrow 0$ as $R \rightarrow \infty$ and that the energy loss rate approach the standard value for a thin disc $\ell_{\text {out }}^{2}-b_{\text {out }}$ per unit mass flow. We anticipate that this energy loss will change from outflow to radiation with increasing radius, though we do not need to specify how rapidly this occurs. We do require the flows of mass, momentum and energy, $\dot{M}, F_{L}$ and $F_{E}$, to vary monotonically with radius; this does restrict the types of function that we choose. (Note that there is a constant positive angular momentum flux, $F_{L, \text { out }}$, flowing inward through the outer disc.) However, there is still considerable freedom 

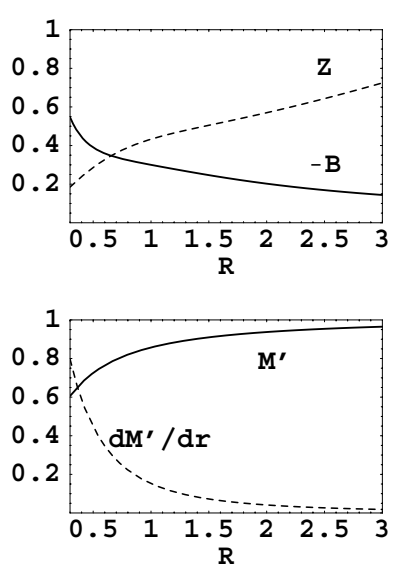
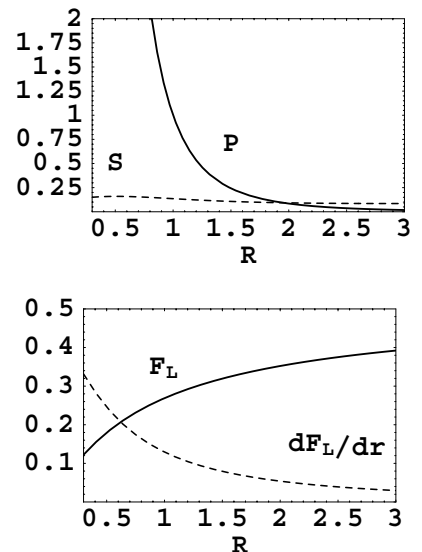
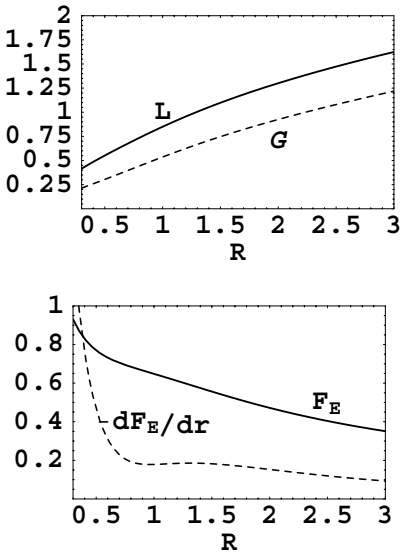

Figure 9. Model of the transition disc that connects the self-similar disc to an outer thin disc. The inner disc is chosen to have the parameters of fiducial model I. The outer disc is a much thinner self-similar disc with $L(R)=0.95 R^{1 / 2}, B(R)=-0.45 R^{-1}$. The top three panels show the variation of the Bernouilli function $B$ and the disc thickness $Z=\left(P / \bar{\rho} \Omega^{2}\right)^{1 / 2}$, the pressure $P$ and the entropy $S$, the angular momentum $L$ and the torque $G$. The bottom three panels show the flows of mass, angular momentum and energy through the disc, and the associated outflows per unit radius.

left and so we do not believe that these solutions are particularly contrived.

Without loss of generality we set the mass supply rate from the outer disc and the pressure at $R=1$, in the middle of the transition region, to unity. We also simplify matters by setting $\eta=0$. Our interpolating functions are:

$$
\begin{aligned}
L(R) & =\frac{\left\{\ell_{\mathrm{out}}+\ell_{0}+\left(\ell_{\mathrm{out}}-\ell_{0}\right) \tanh [\ln R / w]\right\} R^{1 / 2}}{2} \\
B(R) & =\frac{b_{\text {out }}+b_{0}+\left(b_{\text {out }}-b_{0}\right) \tanh [\ln R / w]}{2 R} \\
F_{L}(R) & =\frac{2 n \ell_{0} R^{1 / 2} \dot{M}}{(1+2 n)\left\{1+\left[\frac{2 n \ell_{0}}{(1+2 n) F_{L, \text { out }}}\right]^{2} R\right\}^{1 / 2}}
\end{aligned}
$$

and $\mathcal{G}$ is given by equation (6). After some experimentation, we find that the choices $w=0.8, F_{L, \text { out }}=0.5$ lead to plausible solutions. It is straightforward to solve for the speed of sound, pressure, density and entropy function using equation (1) and integrating the radial equation of motion. We then solve for $\dot{M}$ by integrating equation (5) and $F_{E}(R)$ using equation (8). Our results for this particular example are exhibited in Fig. 9.

These one-dimensional results can be used to verify that the disc remains stable according to the first Høiland criterion. If the disc remains gyrentropic, and the case for this weakens as it becomes increasingly radiative, then it is straightforward to repeat the analysis carried out in Section 6.2 and construct a two-dimensional model. The gyrentropes remain close to right cylinders in the transition disc.

\subsection{Choice of disc-outflow model}

We argued in Section 2.1 that three internal parameters that characterize self-similar discs, which we chose to be $\ell_{0}, b_{0}$ and a torque parameter, determine the nature of the outflow, as measured by $n, \beta$ and $\eta$. How is this linkage established in practice? A comprehensive answer to this question must await more numerical experiments but some qualitative guidelines can be uncovered using our models. The first point is that angular momentum and mass are supplied to the adiabatic flow at the transition radius and are lost as the gas flows inward. Likewise, the energy derives from the relativistic regime and flows outward. The ratio of angular momentum flux to supplied mass flux in the transition region, $F_{L} / \dot{M}$, which is presumably set by local and outer boundary conditions, can have an influence on the subsequent inflow. If this ratio is large, then the disc will rotate rapidly and this can be propagated inward. Likewise, the energy per unit accreted mass $-F_{E} / \dot{M}$ - emerging from the relativistic inner disc controls the disc pressure and thickness and this can propagate outward.

However, this is not the whole story. The local physics within the self-similar regime is also important. If the rate of production of entropy near the disc surface is high, then mass loss will increase. Conversely, when it is low there will be more internal circulation to larger radii, where energy can escape more easily. If the disc has an organized magnetic field, then the loss of angular momentum per unit mass will be larger and the internal angular momentum transport will be diminished. Mathematically, we should think of coupled differential equations for the flows of mass, energy and angular momentum that have to be solved with boundary conditions at both small and large radius, just as with the theory of stellar structure.

\section{ALTERNATIVE MODELS OF ADIABATIC, FLUID ACCRETION}

\subsection{Non-convective models}

In an alternative description of thick accretion discs, pioneered by Paczyński \& Abramowicz (1982) for radiation-dominated tori, (cf. Rees et al. 1982 for the ion-dominated case), the flow is idealized as a quasi-stationary torus orbiting the black hole, in hydrostatic equilibrium with an ad hoc entropy distribution that is commonly chosen to be barytropic, $P=P(S)$. If this flow were convectively stable, the entropy would have to rise vertically as there can be no rotational stabilization when a Høiland interchange is performed in the vertical direction. As there is a pressure maximum, and the isentropes coincide with the isobars in a barytropic flow, the isentropes would have to be a set of nested tori with minimum entropy at the pressure maximum.

However, this configuration cannot be stationary. To see this, note that the conservation laws, equations (52) and (56) (with $Q=0$ ), combine to give the entropy conservation law

$\boldsymbol{G} \cdot \nabla \Omega+H \boldsymbol{J} \cdot \nabla \ln S=0$. 
Now, with a local torque, the first term in equation (99) will be negative, consistent with the second law of thermodynamics (cf. Landau \& Lifshitz 1959). This implies that $\boldsymbol{J} \cdot \nabla \ln S>0$, i.e. there must be a mass flux directed toward increasing entropy. However, this would mean that there must be a mass source $(\nabla \cdot \boldsymbol{J} \neq 0)$ at the pressure maximum. It is therefore clear that convectively stable, barytropic tori cannot be stationary. This conclusion is not altered by the inclusion of general relativity and is probably generally true for two-dimensional, Høiland-stable disc flows, although we have not been able to give a proof. Typically, what will happen is that any initially stable flow will quickly become convective, due to the action of the viscous stress, and the topology of the isentropes will change so that the entropy decreases with increasing cylindrical radius.

\subsection{Advection-dominated accretion flows}

As outlined in Section 1, the original ADAF idea (Ichimaru 1977; Narayan \& Yi 1994, 1995) was that the inflow would be conservative with all the mass supplied at large radius flowing across the horizon. This would probably be the case as long as there were no angular momentum. However, as soon as the inflow is controlled by viscous torque that can be treated perturbatively, there seems no escape from the conclusion that most of the mass supplied escapes in an outflow, powered by the energy released by the small fraction of the gas that accretes on to the hole. (It is, in principle, possible that the viscous torques could be strong enough to ensure radial inflow, which would vitiate this conclusion, but there is strong analytical and numerical evidence that neither fluid nor magnetofluid torques could ever be this large.) At a global level, the physical inconsistency is manifested in a positive Bernouilli function, indicating that parcels of matter near the surface of the disc can escape to infinity with positive energy after doing work on their surroundings. At a local level, the positive energy condition implies convective instability (in the hydrodynamic limit), providing a framework for understanding the mechanism of energy, angular momentum, and mass transport. In Paper II, we will show that analogous arguments carry over to magnetohydrodynamic discs, despite the fact that the stability and transport mechanisms are likely to be quite different.

For these reasons, we argue that black holes surrounded by radiatively inefficient flows are likely to accrete matter at a rate far smaller than the rate at which mass is supplied at large radii. The gas density close to the hole, from where most of the observed emission derives, is therefore likely to be orders of magnitude smaller than derived under the ADAF framework, implying that interpretations of observational data within the latter framework are incorrect. We note, however, that the ADIOS framework developed in this paper does not automatically predict the relationship between the accretion rate and the supply rate. This depends not only on additional information concerning the outer supply (or 'transition') radius, but also on the microphysical mechanisms that increase the entropy and power the wind at the thermal front. [In particular, the magnetohydrodynamic, (MHD) winds to be discussed in Paper II may be capable of carrying away the required energy and angular momentum with very little loss of mass.]

\subsection{Convection-dominated accretion flows}

The CDAF idea was developed to address the energetic difficulties encountered by ADAFs (Narayan, Mahadeven \& Quataert 1998; Narayan et al. 2000; Quataert \& Gruzinov 2000). These models are based on the surprising fact that fluid dynamical turbulence, as might arise, for example, from convection, is capable of transporting angular momentum radially inward in an accretion disc (cf. Ryu \& Goodman 1992; Balbus 2000; Quataert \& Chiang 2000). In CDAF models, it is supposed that the inward convective stress essentially balances the outward viscous stress locally. Consequently, both the mass flux and the net angular momentum flux, which have different natural scalings with radius, must vanish while the energy flux is directed outward. In the two-dimensional model of Quataert \& Gruzinov (2000), $B \sim 0$ everywhere and the flow must extend all the way to the pole. More detailed models assume that $B$ is very small, of the order of the binding energy at the outer (or transition) radius, and that a small conservative accretion flow is responsible for powering the outward flux of energy. The latter is assumed to be disposed of (e.g. by radiation or slow outflow) far from the black hole.

As convective transport is essentially non-local, there is no thermodynamic objection to the average convective torque being negative, although there may be thermodynamic limitations on the extent to which the (macroscopic) convective stress can cancel the (microscopic) viscous stress locally (Balbus \& Hawley 2002). However, the invocation of radial convective transport does seem arbitrary, given the nature of the convective instability. Recall that, at marginal stability in the hydrodynamic (non-MHD) limit, the unstable motions are along the gyrentropes, along roughly spherical surfaces. Radial modes are highly stable. Now if there is no means of extracting energy from the disc surface or the disc extends to the poles, then there will be no net transport and the disc may be forced toward a state in which radial convection can develop. This tendency may be present in numerical simulations by SPB99, some of which show large mass circulations with very small net mass flux.

Convection along gyrentropes is explicitly suppressed, for example, in the CDAF model by Quataert \& Gruzinov (2000), which extends all the way to the polar axis. We believe that this model is physically implausible for two reasons. The first is that flows that do not contain a central funnel have a singular velocity along the axis $v_{\phi}(0)=1$. The rates of shearing, dissipation and entropy production near the axis will therefore diverge, driving a powerful outflow, which creates a funnel (cf. BB99). Because of the symmetry at this point, the viscous stress at the pole cannot be balanced by convective stresses. The second reason is that, unlike with the case of accretion on to a star, there is no means of supporting a column of gas along the rotation axis above a black hole: the centrifugal force acts along a perpendicular direction to the gravity and the pressure force must vanish close the event horizon of the hole (cf. Narayan, Kato \& Honma 1997, BB99). Once the funnel exists, we assert that convection will preferentially operate along gyrentropes, leading to mass and energy loss from the funnel walls.

CDAFs also face secular difficulties related to the global mass supply and energy flow. In CDAFs, as in ADIOS models, the accretion rate reaching the black hole is far smaller than the mass supply at large radii. However, unlike ADIOS models, there is no means of escape for the vast majority of the supplied matter, which does not make it to the black hole. In the hydrodynamical simulations by SPB99 and others, this is not an issue because these calculations track the evolution of a finite torus; matter is not continuously supplied. (Moreover, the runs are not long enough to track the evolution of the outer flow.) However, in a CDAF with a continuous mass supply, there is no alternative but to establish an increasing reservoir of matter that is unable to accrete. Thus, CDAFs cannot represent steady-state flows. Moreover, there is a continuous flow of energy into the outer parts of a CDAF, where it will also build up unless there is some escape route. It has been proposed that the outer 
parts of ADAFs may adjust to radiate away this energy (Abramowicz \& Igumenshchev 2001; Ball, Narayan \& Quataert 2001), but it has not been demonstrated that this will happen naturally. Another possibility is that the energy flux will power a wind at some outer radius. We contend that this too is a less likely resolution than a scheme in which the wind is released continuously from all radii.

Even if CDAFs did not face these severe physical problems, their existence seems unlikely to carry over to the MHD case, which is more appropriate to astrophysical discs than the hydrodynamical limit. The dominant instabilities in magnetized discs are magnetorotational, rather than convective, and the resulting turbulence is likely to transport angular momentum outward rather than inward (Balbus \& Hawley 2002); but see Igumenshchev (2002); Narayan et al. (2002) for an alternative view. If this is the case, then the CDAF approach cannot be generalized to MHD flows. However, we will argue in Paper II that the ADIOS approach does carry over. Although MHD discs cannot attain marginal stability, they nevertheless appear to develop a well-defined internal structure that provides an equivalent autonomous constraint to our hydrodynamical assumption of gyrentropicity. Thus, MHD discs admit well-defined circulation patterns and outflows, which we will derive explicitly in Paper II.

\subsection{Weakly bound dises}

Finally, we comment on a class of non-radiative accretion models most recently studied by Paczyński (1998), but which enjoyed wide popularity in the early 1980s (e.g. Jaroszyński, Abramowicz \& Paczyński 1980; Paczyński \& Wiita 1980 and references therein). In these models the angular momentum distribution is prescribed, usually as a function of cylindrical radius along the surface of the flow, between some inner radius $r_{\text {in }}$ and some outer radius $r_{\text {out }}$. At $r \geqslant r_{\text {out }}$ the flow is assumed to match a thin Keplerian accretion disc, while at $r \rightarrow r_{\text {in }}$ the flow approaches a zero-pressure cusp through which material accretes on to the black hole. For the interesting limit of $r_{\text {out }} \gg r_{\text {in }}$ the gas in the advection-dominated region is very weakly bound and one can show (Paczyński 1998) that the cusp must lie close to the marginally bound orbit at $r=4 m$ (for a Schwarzschild black hole). There is considerable latitude in the choice of angular momentum distribution, subject to the constraints that the angular momentum must be a monotonically increasing function of $r$, and that the angular momentum and binding energy must match the Keplerian values at both $r_{\text {in }}$ and $r_{\text {out }}$.

One of the rationales for exploring these models is that the generic form of the angular momentum distribution is physically motivated, whereas viscous stress prescriptions are highly uncertain. It is therefore worthwhile, Paczyński argues, to construct a model based on an assumed angular momentum law, and then see what kind of viscous stress prescription is required to make it self-consistent. However, in his published work he does not discuss the nature of these stress prescriptions. We now show that weakly bound disc models require very specific and (we believe) implausible prescriptions for the viscous stress.

We restrict our attention to the limit $r_{\text {out }} \gg r_{\text {in }}$ and use the notation in Paczyński (1998). Paczyński (1998) equation (25) implies that the binding energy is a monotonically increasing function of $r_{\mathrm{s}}$, the cylindrical radius measured along the surface. This implies the inequality

$\int_{r_{\text {in }}}^{r_{\mathrm{s}}} \frac{1}{r^{2}} \frac{\mathrm{d} j_{\mathrm{s}}^{2}}{\mathrm{~d} r} \mathrm{~d} r<\frac{2 j_{\text {out }}^{2}}{r_{\text {out }}^{2}} \approx \frac{1}{2 r_{\text {out }} r_{\text {in }}} j_{\text {in }}^{2}$ for all $r_{\text {in }}<r_{\mathrm{s}}<r_{\text {out }}$. Integrating by parts, and using the inequality $j_{\mathrm{s}} \geqslant j_{\text {in }}$, we finally obtain

$j_{\mathrm{s}}^{2}<j_{\text {in }}^{2}\left(1+\frac{r_{\mathrm{s}}^{2}}{2 r_{\mathrm{out}} r_{\mathrm{in}}}\right)$.

Thus, the angular momentum distribution must be a very weak function of radius, $j \approx j_{\text {in }}$ for $r_{\mathrm{s}}$ smaller than the geometric mean between $r_{\text {in }}$ and $r_{\text {out }}$. In other words, any acceptable angular momentum distribution for this kind of model must be nearly constant in the inner part of the disc, while increasing more steeply than Keplerian in the outer parts (in order to satisfy the outer boundary condition).

Inequality (101) places severe constraints on the viscous stress. Using the $\alpha$-model viscous couple assumed by Afshordi \& Paczyński (2002), which differs from our assumed torque (equation 49) by a factor $\Omega / \Omega_{\mathrm{Kep}}$, and assuming an internal speed of sound $v_{\mathrm{s}} \sim v_{\text {Kep }}$ (appropriate for a thick disc), we obtain an inflow speed $v_{r}$ given by

$\frac{-v_{r}}{v_{\text {Kep }}} \approx \alpha \frac{j_{\mathrm{s}}}{j_{\mathrm{s}}-j_{\text {in }}}\left(-\frac{\mathrm{d} \ln \Omega}{\mathrm{d} \ln r}\right)$.

Substituting from inequality (101) in the limit $r_{\mathrm{s}} \ll\left(r_{\text {in }} r_{\text {out }}\right)^{1 / 2}$, we can write equation (102) in the form

$\alpha<\frac{r_{\mathrm{s}}^{2}}{8 r_{\text {in }} r_{\text {out }}} \frac{-v_{r}}{v_{\text {Kep }}}$.

The hydrostatic condition implies $-v_{r} / v_{\text {Kep }} \ll 1$, placing a tight upper limit on $\alpha$. The limit is even more severe if we use our expression (49) for the stress.

If $\alpha$ exceeds the limit given in equation (103), over any range of radii, then the inflow will proceed on a free-fall time-scale, violating a principal assumption of the model. This will presumably continue until the angular momentum distribution relaxes to state closer to Keplerian, in which case it will resemble one of the models discussed above (i.e. an ADAF, CDAF, or ADIOS). It will also evolve toward a larger binding energy. We would, of course, argue that the only self-consistent final state under these circumstances would be an ADIOS.

Physically, we expect the value of $\alpha$, or its equivalent in a more realistic stress model, to be set by the microphysics of the accretion process and not by the global boundary conditions - the latter presumably reflect the cooling function of the gas and/or the nature of the gas supply at $r_{\text {out }}$. Numerical models of MHD flows suggest that $\alpha$ may fall in the range $\sim 0.01-0.1$, which is inconsistent with the limit derived above unless $r_{\text {out }} \lesssim 100 r_{\text {in }}$. Observations of adiabatic accretion flows suggest that $r_{\text {out }}$ may be larger than $\sim 10^{4}-10^{5}$ times $r_{\text {in }}$. Such systems would have to have an effective $\alpha<10^{-5}$ in order to be described self-consistently by a Paczyński model. Thus, we conclude that weakly bound disc models are unlikely to apply in many, if not most, cases of astrophysical interest.

\section{DISCUSSION}

We have attempted to flesh out the ADIOS concept by constructing explicit, two-dimensional models of adiabatic, accreting fluid. Such flows are strongly convective and should naturally evolve toward a state of marginal stability. We argue that the convective transport of energy and angular momentum does not occur primarily in the radial direction but, instead, proceeds primarily along surfaces that connect the equatorial region to the disc 'surface' at high latitudes. We have demonstrated how to construct models of two-dimensional discs embodying these principles, and have then elaborated upon 
them so as to include poloidal circulation, inflow and the formation of fluid outflows.

Even in the context of purely fluid discs, our approximate description of the flow can be challenged. For example, if the viscous stress, as measured by the parameter $\alpha$, is not small, the perturbative ordering: circular speed $\gg$ convective speed $\gg$ meridional circulation/inflow speed will not be well-satisfied as we require. Our thermal front model for producing the outflow is, likewise, an oversimplification. It posits a site where entropy is produced and simple conservation laws make explicit the connection between the energy flow along the gyrentropes and the outflow. It seems reasonable that the dissipation should be a strongly increasing function of the Mach number and that it be efficient when the flow becomes roughly sonic but this is not required. If the surface dissipation is small, we suppose that the energy will be advected by the circulation to large radius. Indeed, as discussed in Section 3.2, several simulations do seem to show this behaviour, which is not consistent with a self-similar flow. Higher-resolution simulations that are run for long enough to achieve a stationary flow will be needed to understand the flow of mass, angular momentum and energy through the disc.

Another concern is that our calculation is expressly twodimensional and it is well known that three-dimensional convection in non-rotating fluid is quite different from its two-dimensional counterpart. However, if our convection model is realistic, it automatically leads to considerable smoothing over azimuth, which ought to validate the two-dimensional description. A related concern involves the unstable, non-axisymmetric, global instabilities that can develop in the relativistic inner disc described in Section 6.2 (Papaloizou \& Pringle 1984). As these modes rely upon reflection from the inner surface of the disc, they are unlikely to grow to a large amplitude in the presence of inflow (Begelman, Blandford \& Rees 1984; Blaes 1987).

The most fundamental limitation concerns the explicit neglect of magnetic field in our model. Beyond all reasonable doubt, the torque in astrophysical accretion discs is hydromagnetic in origin (Balbus \& Hawley 1998). If the magnetic fields remained small in strength and length-scale and were locally dissipative, then our treatment could still have validity. However, the evidence from numerical simulations is that none of these conditions is well-satisfied in practice. In particular, the relationship between the momentum transfer and the dissipation is problematic. With a simple, Newtonian viscosity, the connection is clear. There is a local dissipation at a rate $\boldsymbol{G} \cdot \nabla \Omega$. Entropy is created where the torque is applied. The introduction of convection into fluid models complicates this linkage, because it involves the net bodily transfer of mass carrying its own angular momentum and energy and transporting an additional energy flux. This effectively makes the dissipation non-local. As we shall discuss in Paper II, magnetized discs may provide even more extreme examples of non-local dissipation. Unless a magnetic turbulence spectrum is established and the energy cascades down to a small inner scale where it can be taken up by plasma, much of the dissipation may be non-local. Indeed, there is plenty of evidence that much of the dissipation in observed discs occurs in a hot corona. (In considering this problem it is important to make the distinction between ion-supported and radiation-supported discs. The former require that local dissipation not heat the electrons to temperatures where they can radiate efficiently, implying either that most of the energy either goes into heating the ions or that the energy is transported away. In contrast, radiation-dominated accretion discs are subject to the magnetorotational instability but the magnetic stress may saturate at a lower level, perhaps validating a treatment closer to our fluid models.)
The final missing ingredient is an allowance for global time dependence in the flow. There are strong indications that observed discs accrete episodically. Whether or not this happens in practice, on all radial scales, depends upon the details of the transition region. In Section 6.3, we argue that there could be a smooth, stationary transition from a radiative thin disc to an ADIOS. However, if the outflow is less efficient than we have assumed in carrying off the energy, circulation of the excess energy to the transition region will push it outward to progressively larger radii until there is catastrophic cooling so that the thick disc quickly shrinks. The whole pattern can repeat in a limit cycle. However, as the inflow and dynamical time-scales are always likely to increase with radius, these variations should only cause a slow secular change in the flow pattern at small radii, where most of the accretion energy is released. In the language of our models this would be manifested in a change in the entropy scale $s_{0}$ (cf. Section 4.3). The other way in which time dependence could be important is if there are local instabilities in the flow pattern. In the case of adiabatic accretion discs the distinction between viscous and thermal time-scales is blurred. This, coupled with the physical thickness of the disc, makes a formal instability calculation more complicated and we advocate direct numerical simulation as the means to explore stability of these flows. Few simulations have been evolved long enough to draw strong conclusions concerning time dependence.

We believe that the fluid dynamical analysis is valuable because it suggests a 'modular' procedure for analysing numerical simulations of magnetized flows.

(i) Determine the rule that replaces gyrentropicity for determining the time-averaged disc structure.

(ii) Separate the motion into relatively small-scale interchanges - with scales no larger than the local pressure scaleheight, and larger circulatory flows. The former can be analysed by computing correlation functions involving velocity, pressure, density, etc, up to third order so as to understand the time-averaged transport of mass, angular momentum and energy as a function of position. The latter will describe the global flow pattern.

(iii) Understand the surface boundary condition, specifically the strength and character of the MHD wind from the disc surface. Central to answering this question is to decide whether or not largescale magnetic fields are generated, as is observed in the high latitude wind from the quiet sun, or whether the fields are tangled on small scale and behave as an anisotropic gas. This problem will be very hard to tackle as it combines both local and global features.

We shall address these issues further in a future paper.

Despite all of these shortcomings and concerns, we hope that the models developed in this paper will provide a guide to interpreting numerical simulations and, ultimately, the increasingly detailed observations of radiation from adiabatically accreting black holes and neutron stars.

\section{ACKNOWLEDGMENTS}

We are indebted to many colleagues, notably Omer Blaes, Martin Rees and Jim Stone, for advice and encouragement. This work was supported in part by NSF grants AST-9529170, AST9529175, AST-9876887 and AST-0307502, NASA grants NAG 52837 and NAG5-7007 and the US Department of Energy under contract number DE-AC03-76SF00515. Much of the research reported here was carried out during 1999-2000 at the Institute of Astronomy at the University of Cambridge; the Kavli Institute for Theoretical Physics at the University of California, Santa Barbara; and the 
Institute for Advanced Study. We thank the members of these institutes for their hospitality.

\section{REFERENCES}

Abramowicz M.A., Igumenshchev I.V., 2001, ApJ, 554, L53

Abramowicz M.A., Chen X., Kato S., Lasota J.P., Regev O., 1995, ApJ, 438, L37

Abramowicz M.A., Lasota J.-P., Igumenshchev I., 2000, MNRAS, 314, 775

Afshordi N., Paczyński B., 2002, preprint, astro-ph/0202409

Agol E., 2000, ApJ, 538, L121

Aitken D.K. et al., 2000, ApJ, 530, 173

Baganoff F.K. et al., 2003, ApJ, 591, 891

Balbus S.A., 2000, ApJ, 534, 420

Balbus S.A., Hawley J.F., 1998, Rev. Mod. Phys., 70, 1

Balbus S.A., Hawley J.F., 2002, ApJ, 573, 749

Ball G.H., Narayan R., Quataert E., 2001, ApJ, 552, 221

Bardeen J.M., 1973, in DeWitt C., DeWitt B., eds, Black Holes. Gordon and Breach, New York

Bardeen J.M., Berger B.K., 1978, ApJ, 221, 105

Begelman M.C., 1979, MNRAS, 187, 237

Begelman M.C., 2002, ApJ, 568, 97

Begelman M.C., Meier D.L., 1982, ApJ, 253, 873

Begelman M.C., Blandford R.D., Rees M.J., 1984, Rev. Mod. Phys., 56, 255

Blaes O.M., 1987, MNRAS, 227, 975

Blandford R.D., 1984, in Evans D.S., ed., 11th Texas Symp. on Relativistic Astrophysics. Ann. NY Acad. Sci., 422, p. 303

Blandford R.D., Begelman M.C., 1999, MNRAS, 303, L1 (BB99)

Blandford R.D., Payne D.G., 1982, MNRAS, 199, 883

Blandford R.D., Jaroszyński M., Kumar S., 1985, MNRAS, 215, 667

Bower G.C., Wright M.C., Falcke H., Backer D.C., 2003, ApJ, 588, 331

Di Matteo T., Fabian A.C., Rees M.J., Carilli C.L., Ivison R.J., 1999, MNRAS, 305, 492

Di Matteo T., Quataert E., Allen S.W., Narayan R., Fabian A.C., 2000, MNRAS, 311, 507

Fabian A.C., 2003, in Ho L., ed., Carnegie Observatories Astrophysics Series, Vol. 1, Coevolution of Black Holes and Galaxies. Cambridge Univ. Press, Cambridge, in press (astro-ph/0304122)

Frank J., King A.R., Raine D.J., 2001, Accretion Power in Astrophysics. Cambridge Univ. Press, Cambridge

Goldreich P., Schubert G., 1967, ApJ, 150, 571

Hansen C.J., Kawaler S.D., 1994, Stellar Interiors: Physical Principles, Structure, and Evolution. Springer, Berlin

Ichimaru S., 1977, ApJ, 214, 840

Igumenshchev I.V., 2002, ApJ, 577, 31
Jaroszyński M., Abramowicz M.A., Paczyński B., 1980, Acta Astr., 30, 1

Kato S., Nakamura K.E., 1998, PASJ, 50, 559

King A.R., Taam R., Begelman M.C., 2000, ApJ, 530, L25

Klahr H.H., Bodenheimer P., 2003, ApJ, 582, 869

Königl A., Pudritz R.E., 2000, in Mannings V., Boss A.P., Russell S.S., eds, Protostars and Planets IV. Univ. Arizona Press, Tucson, p. 759

Landau L.D., Lifshitz E.M., 1959, Fluid Mechanics. Pergamon, Oxford

Manmoto T., Kato S., Nakamura K.E., Narayan R., 2000, ApJ, 529, 127

Margon B., 1984, ARA\&A, 22, 507

Melia F., Falcke H., 2001, ARA\&A, 39, 309

Mirabel I.F., Rodriguez L.F., 1999, ARA\&A, 37, 409

Mushotzky R., Angelini L., Arnaud K.A., Loewenstein M., 2000, AAS High Energy Astrophysics Division, 32, 2101

Narayan R., Yi I., 1994, ApJ, 428, L13

Narayan R., Yi I., 1995, ApJ, 444, 231

Narayan R., Kato S., Honma F., 1997, ApJ, 476, 49

Narayan R., Mahadevan R., Quataert E., 1998, in Abramowicz M.A., Bjornsson G., Pringle J.E., eds, The Theory of Black Hole Accretion Disks. Cambridge Univ. Press, Cambridge, p. 148

Narayan R., Igumenshchev I.V., Abramowicz M.A., 2000, ApJ, 539, 798

Narayan R., Quataert E., Igumenshchev I.V., Abramowicz M.A., 2002, ApJ, 577, 295

Paczyński B., 1998, Acta Astr., 48, 667

Paczyński B., Abramowicz M.A., 1982, ApJ, 253, 897

Paczyński B., Wiita P., 1980, A\&A, 88, 23

Papaloizou J.C.B., Pringle J.E., 1984, MNRAS, 208, 721

Quataert E., Chiang E.I., 2000, ApJ, 543, 432

Quataert E., Gruzinov A., 2000, ApJ, 539, 809

Quataert E., Narayan R., 1999, ApJ, 520, 298

Rees M.J., Begelman M.C., Blandford R.D., Phinney E.S., 1982, Nat, 295, 17

Ryu D., Goodman J., 1992, ApJ, 388, 438

Seguin F., 1975, ApJ, 197, 745

Shakura N.I., Sunyaev R.A., 1973, A\&A, 24, 337

Soltan A., 1982, MNRAS, 200, 115

Stone J.M., Pringle J.E., 2001, MNRAS, 322, 461

Stone J.M., Pringle J.E., Begelman M.C., 1999, MNRAS, 310, 1002 (SPB99)

Tassoul J.-L., 1978, Theory of Rotating Stars. Princeton Univ. Press, Princeton

Weymann R., 1997, in Arav N., Shlosman I., Weymann R.J., eds, Mass

Ejection from AGN. Astron. Soc. Pacific, San Francisco, p. 3.

Yu Q., Tremaine S.D., 2002, MNRAS, 335, 965

This paper has been typeset from a $\mathrm{T}_{\mathrm{E}} \mathrm{X} / \mathrm{L} \mathrm{T}_{\mathrm{E}} \mathrm{X}$ file prepared by the author. 